\title{
Serglycin-Mediated Selective Reactivation of the Novel YAP/CRISPLD2 Developmental Axis Promotes Metastasis and Sorafenib Resistance in Hepatocellular Carcinoma
}

\section{HAO HU}

Sun Yat-sen University First Affiliated Hospital

\section{Liang $\mathrm{Xu}$}

Sun Yat-sen University Sixth Affiliated Hospital

Tie-Jun Huang

Shenzhen Second People's Hospital

Fei-Fei Luo

Sun Yat-sen University Affiliated Tumor Hospital: Sun Yat-sen University Cancer Center Jing Wang

Sun Yat-sen University Cancer Center

\section{Yan Mei}

Sun Yat-sen University Cancer Center

\section{Li-Sheng Zheng}

Sun Yat-Sen University Cancer Prevention and Treatment Center: Sun Yat-sen University Cancer Center

\section{Li-Xia Peng}

Sun Yat-Sen University Cancer Prevention and Treatment Center: Sun Yat-sen University Cancer Center

\section{Dong-Fang Meng}

Shandong First Medical University

\section{Zhan-Fei Zhang}

People's Hospital of Zhongshan

\section{Shi-Jun Zhang}

sun yat-sen university

\section{Chao-Nan Qian}

sun yat-sen university of cancer center

Bi-Jun Huang ( $\sim$ huangbj@sysucc.org.cn )

Sun Yat-sen University Affiliated Tumor Hospital: Sun Yat-sen University Cancer Center

\section{Research}


Keywords: Hepatocellular carcinoma, Serglycin, metastasis, YAP pathway, CRISPLD2

Posted Date: December 29th, 2020

DOI: https://doi.org/10.21203/rs.3.rs-135613/v1

License: (c) (1) This work is licensed under a Creative Commons Attribution 4.0 International License. Read Full License 


\section{Abstract}

Background. The extracellular matrix (ECM) component serglycin promotes the epithelial-tomesenchymal transition (EMT) and metastasis in an autocrine manner. However, the detailed mechanism underlying the roles of serglycin in Hepatocellular carcinoma (HCC) metastasis progression remains to be clarified.

Methods: Analyzing 123 patients' serum by ELISA to investigate the association between serglycin expression and HCC metastasis and prognosis. Serglycin, CRISPLD2, and YAP were overexpressed or knocked down with vector or RNAi. The RNA - sequence was used to screen serglycin downstream effectors, followed by bioinformatics, ChIP-PCR, luciferase, and promoter site mutation to identify novel target genes. The metastatic abilities of serglycin were demonstrated by a series of in vitro and in vivo experiments. Immunofluorescence, flow cytometry, and western blotting were carried out to demonstrate the potential mechanisms of serglycin.

Results: We observed that serglycin was overexpressed in HCC tissues and serum, and its level was associated with metastasis and poor prognosis. By gain- and loss-of-function approaches, we found serglycin affects migratory motility and metastatic capacity in vitro and in vivo, and it's significantly associated with the stemness-like properties. Interestingly, serglycin activated the effector YAP of Hippo pathway, and further study verified the crucial role of serglycin/YAP in tumorigenesis by the DEN-induced HCC mouse model. Furthermore, the CRISPLD2 was selectively upregulated by serglycin, the axis of serglycin / YAP / CRISPLD2 regulated metastatic capacity in HCC cells, it revealed CRISPLD2 was the direct target gene of serglycin-mediated YAP/TEAD1 complex. Besides, serglycin-mediated YAP pathway enhanced cell sorafenib resistance, sorafenib combined with verteporfin reversed serglycin-mediated cellular insensitivity to sorafenib therapy.

Conclusions. Autocrine ECM serglycin plays a crucial role in the process of stemness maintenance, tumorigenesis, and metastasis via selective reactivation of the novel YAP/CRISPLD2 developmental axis promotes metastasis and sorafenib resistance in Hepatocellular carcinoma, YAP-TEAD1 inhibitor verteporfin reversed serglycin-mediated cellular sorafenib resistance.

\section{Introduction}

Hepatocellular carcinoma (HCC) is the sixth most prevalent cancer and third most frequent cause of cancer-related death [1]. Additionally, HCC is the predominant pathological type of liver cancer, accounting for more than $80 \%$ of liver cancer cases [2]. Each year, hepatocellular carcinoma is diagnosed in more than half a million people worldwide. Much research has focused on diagnostic strategies to identify early HCC, and the diagnostic tools commonly used include the serum tumor marker alphafetoprotein (AFP), carcinoembryonic antigen (CEA), radiographic imaging, and liver biopsy. Serum AFP used alone can be helpful if the levels are markedly elevated, a condition that occurs in fewer than half of cases at the time of diagnosis, but its sensitivity and specificity are limited. Therefore, it is necessary to 
investigate specific biomarkers involved in the metastatic process at the gene expression and serum level.

Furthermore, most HCC-associated deaths are caused by metastatic disease rather than primary tumors $[3,4]$. It has important functions in the formation of several types of storage granules and comprises a 17.6-kDa core protein to which eight negatively charged glycosaminoglycan (GAG) chains of either chondroitin sulfate or heparin are attached [5]. The core protein containing 158 amino acid residues can be divided into 3 domains: a signal peptide domain, an $\mathrm{N}$-terminal domain with unknown function, and a C-terminal domain [6]. Although SRGN does not contain a transmembrane domain, it can be constitutively secreted by hematopoietic cells, endothelial cells, pancreatic acinar cells, and myeloma cells [7-10]. Moreover, SRGN has been documented to be a secretory product and can easily incorporate into the ECM or associate with the surfaces of target cells [11-13]. In our previous study, we first reported that SRGN promotes nasopharyngeal carcinoma (NPC) epithelial-to-mesenchymal transition (EMT) and metastasis in an autocrine manner $[11,14]$, but the underlying mechanism of SRGN in HCC remains unknown.

In past decades, the Hippo/YAP pathway has been delineated and shown to play an important role in animal organ size control, which it exerts by regulating tissue proliferation and apoptosis rates as a response to developmental cues, cell contact, and density[15]. Yes-associated protein (YAP) is the major downstream effector of the Hippo pathway, and, not surprisingly, it functions as an oncogene. The YAP gene is amplified in several human cancers[16], and increased YAP expression and nuclear localization have been observed in liver cancers, colon cancers, ovarian cancers, lung cancers, and prostate cancers $[17,18]$. YAP expression and nuclear localization strongly correlate with poor patient outcomes and several tumor progression [19]. YAP1 activation can reversibly increase liver size more than 4-fold, and it is highly expressed in stem cells [20]. In the intestine, YAP1 can stimulate Notch signaling, and the administration of $g$-secretase inhibitors can suppress the intestinal dysplasia caused by YAP1 [21]. However, little is known about the detailed mechanism underlying the relationship between SRGN and HCC progression.

Sorafenib, an oral multikinase inhibitor with anti-proliferative and anti-angiogenic activity, has been considered the standard of care for patients with advanced unresectable hepatocellular carcinoma [22]. Unfortunately, HCC resistance to sorafenib is very common. Cancer stem cells (CSCs) have the properties of self-renewal, differentiation, and resistance to sorafenib therapy or radiotherapy [23]. Although CSCs represent a small proportion of the tumor cell population, they are key players in tumor initiation, recurrence, and metastasis [24]. In HCC cells that highly express SRGN, CSCs activated by YAP can lead to sorafenib resistance, so there is an urgent need to find a new targeted therapy.

\section{Materials And Methods}

\section{Cell Lines and Cell Culture}


The human HCC cell lines SK-Hep-1, MHCC-97H, MHCC-LM3, Hep3B, HepG2, and LO-2 were routinely cultured in Dulbecco's Modified Eagle Medium (DMEM; Gibco; c11995500bt) supplemented with 10\% fetal bovine serum (Invitrogen; 10099141) as well as penicillin (Bioss; bs-10687PA-1). All the cells were grown at $37^{\circ} \mathrm{C}$ in a humidified incubator containing $5 \% \mathrm{CO}_{2}$. Further details are available supplementary methods.

\section{Cell Proliferation Assay}

The colorimetric MTS assay (Cell Titer 96 Aqueous One Solution Cell Proliferation Assay) was performed to determine cancer cell growth and viability as previously reported[25]. Cells (1000 cells/200 $\mu$ l of medium) were seeded in a 96-well plate (Corning; 3599) and cultured under standard conditions. At various time points after seeding, the cells in each well were stained with $20 \mu$ of MTS (Promega; G3580) for $3 \mathrm{~h}$, and the absorbance value 490 (OD490) was determined using a microplate reader. All the experiments were independently repeated at least three times.

\section{Transwell Assay}

For the Transwell migration assay, $3 \times 10^{4}$ cells in $200 \mu$ l of serum-free DMEM was added to the cell culture inserts with an 8- $\mu \mathrm{m}$ microporous filter without extracellular matrix coating (Corning; 3428). Next, $800 \mu \mathrm{l}$ of DMEM medium containing 10\% FBS was added to the bottom chamber. After $20 \mathrm{~h}$ of incubation, the cells in the lower surface of the filter were fixed, stained with $1 \%$ crystal violet (TargetMol, T1343L), and examined. Both experiments were repeated independently three times.

\section{Cytoplasmic and Nuclear Protein Extraction}

Adherent cells were grown to $90-100 \%$ confluence, and the cells were washed twice with cold PBS, followed by aspiration of the buffer. Next, appropriate amounts of cytoplasmic extraction buffer added, followed by swirling to distribute the lysis buffer over the entire surface of tissue cultures and placing the cells on ice for $5 \mathrm{~min}$. The lysed cells were scraped with a pipette tip or a transfer pipette, and the cell lysate was transferred to a pre-chilled $1.5-\mathrm{ml}$ micro-centrifuge tube. The tube was vortexed vigorously for 15 seconds, followed by centrifugation for 5 min at top speed in a microcentrifuge at $4{ }^{\circ} \mathrm{C}$. The supernatant (cytosol fraction) was transferred to a fresh pre-chilled 1.5-ml tube. Appropriate amounts of nuclear extraction buffer were added to the pellet, and the mixture was vortexed vigorously for 15 seconds and then incubated on ice for one min. Vortexing was repeated 4 times, 15 seconds each, followed by incubation for one min. The nuclear extract was immediately transferred to a pre-chilled filter cartridge with a collection tube and centrifuged at top speed $(14,000-16,000 \times \mathrm{g})$ in a microcentrifuge for 30 seconds. The filter cartridge was then discarded, and the nuclear extract was stored at $-80{ }^{\circ} \mathrm{C}$ until use.

\section{Luciferase Assay}

After the cultured cells reached 80-95\% confluence, the growth medium was removed and a sufficient volume of phosphate-buffered saline (PBS) was gently applied to wash the surface of the culture vessel. The vessel was swirled briefly to remove detached cells and residual growth medium, and then the rinse solution was completely removed before applying the lysis buffer. Next, the minimum volume of $1 \times$ Luc- 
Lysis II Buffer required to completely cover the cell monolayer was dispensed into each culture well. The culture plates were then placed on a rocking platform or orbital shaker with gentle rocking/shaking to ensure complete and even coverage of the cell monolayer with $1 \times$ Luc-Lysis II Buffer. The culture plates were rocked at room temperature for 10-15 min. The final data = Firefly luciferase / Renilla luciferase. The Transfection and Luciferase assay was performed in duplicate and repeated independently at least three times. The CRISPLD2 promoter sequence is listed in Supplementary 1.

\section{Animal Experiments}

All the animal experiments were performed following the protocols approved by the Research Animal Resource Center of Sun Yat-Sen University (Approval number L102012017005S). All the animal studies were conducted according to the principles and procedures outlined in the guidelines of the Institutional Animal Care and Use Committee at Sun Yat-Sen University Cancer Center. Male athymic mice between 4 and 5 weeks of age were obtained from the Beijing Vital River Laboratory Animal Center (Beijing, China) and maintained under a specific pathogen-free environment.

For the tumor metastasis experiments, all the nude mice were randomly divided into 3 groups of 12 mice each: control, KD\#1 and KD\#2. Tumor cells $(1.5 \times 106$ cells) were suspended in $50 \mu$ l of phosphatebuffered saline (PBS) and intravenously injected into the tail vein of each mouse. The mice were monitored twice per week. The mice were euthanized 8 weeks after the injection of the cancer cells, and distant metastases in the lungs were assessed and counted. The lungs were excised and embedded in paraffin for further study.

For the tumor xenograft experiments, all the cell lines were cultured in a humidified incubator with $7.5 \%$ $\mathrm{CO} 2$ at $37^{\circ} \mathrm{C}$. The cells were detached using $0.05 \%$ trypsin (Invitrogen) and re-suspended for implantation in serum-free media. Next, $5 \times 106$ cells were implanted subcutaneously in a total volume of $0.1 \mathrm{ml} /$ mouse for SK-Hep-1. Tumor growth was monitored twice weekly using bilateral caliper measurements, the tumor volume was calculated, and the mice were randomized into vehicle or treatment groups with an approximate mean start size of $0.2-0.4 \mathrm{~cm} 3$. For pharmacodynamic studies, the mice were randomized at a mean tumor volume of approximately $0.5-0.8 \mathrm{~cm} 3$ using the same randomization criteria as that in the tumor growth inhibition studies. The mice were then treated orally with a single bolus dose of sorafenib at $10 \mathrm{mg} / \mathrm{kg}$ or a vehicle control (DMSO in PBS) every day for 21 days. Verteporfin was dissolved and freshly diluted in 5\% PEG400 and 5\% Tween 80 before treatment and was administered by intraperitoneal injection at $50 \mathrm{mg} / \mathrm{kg}$ every 3 days. The tumors were excised at specific timepoints after dosing and fixed in $10 \%$ buffered formalin.

For the DEN/CCL4 induced-HCC mouse model, 15 specific pathogen-free (SPF) C57BL/6 mice (male; weight, $23 \pm 2 \mathrm{~g}$ ) were purchased from the Animal Experimental Center of the Guangdong province. The mice were injected with $25 \mathrm{mg} / \mathrm{kg}$ of DEN intraperitoneally for 14 days, and another DEN injection was performed for 30 days to induce HCC development; it will take 10 months to develop HCC.

\section{ChIP Assay}


Cells were cross-linked with $1 \%$ formaldehyde at room temperature for $10 \mathrm{~min}$, and then $10 \times$ glycine was added to cross-linked the cells at room temperature for $5 \mathrm{~min}$. Next, $4 \times 10^{6}$ cells were added to $200 \mu \mathrm{l}$ of ChIP buffer per assay plus $5 \mu$ of micrococcal nuclease, and then the mixture was incubated at $37^{\circ} \mathrm{C}$ for $20 \mathrm{~min}$. The cross-linked chromatin was sonicated to generate DNA fragments ranging from 150 to $900 \mathrm{bp}$. The DNA fragments were immunoprecipitated with 1-2 ug of YAP1 and Histone H3 (CST, D2B12) antibody or normal IgG, and then the purified DNA fragments from chromatin immunoprecipitation were subjected to the ChIP-qPCR to identify CRISPLD2 fragments. The primers are listed in supplementary 2 .

\section{Statistical Analysis}

All statistical analyses were performed using the SPSS (version 24) package. Student's t-test was used to compare two independent groups of data, and the data were represented as means \pm S.D. Spearman's correlation (2-tailed) was used to calculate the correlation coefficient ( $r$ ) and the $p$-value between SRGN and CRISPLD2 expression. The significance of several variables for survival was analyzed using the Cox regression model in multivariate analysis. A $p$-value $<0.05$ was considered statistically significant in all cases.

\section{Results}

\section{SRGN is an Independent Prognostic Factor and Potential Serum Biomarker in HCC Patients}

To investigate the role of SRGN in HCC progression, SRGN mRNA expression in primary tissues was analyzed in the GSE364 cohort. SRGN was upregulated in metastatic primary tissues compared with that in non-metastatic primary tissues (Fig. 1A). Additionally, we found that SRGN expression was significantly higher in tissues with metastasis than in those without metastasis in TCGA database (Fig. 1B), a finding that was similar to that in the GSE364 cohort. Moreover, SRGN expression was significantly higher in lymph node metastasis tissues than in non-lymph node metastasis tissues (Fig. 1C). Serum tumor markers were commonly used as a diagnostic method for HCC, and we speculated that SRGN could be another significant biomarker for HCC diagnosis. To determine the significant role of SRGN in HCC progression, we collected 123 serum samples to evaluate the clinical implications of SRGN expression.

To confirm this hypothesis, we performed ELISA and examined SRGN protein expression, which was significantly higher in the serum of patients with HBV than in the serum of patients without HBV (Fig. 1E). The level of SRGN protein in liver cancer patients without metastasis was higher than that in healthy volunteers, and even higher in patients with metastasis (Fig. 1F). SRGN in the serum of HCC patients was positively correlated with diameter of neoplasms (Fig. 1G), AFP (Fig. 1H), clinical stage (Fig. 1I) and T stage (Supplementary Fig. 1A). Therefore, we elucidated that SRGN is a potential serum biomarker and plays a critical role in HCC progression. 
To evaluate the prognostic value of SRGN expression in primary HCC tissues, we searched the TCGA database of SRGN expression in HCC samples. High expression of SRGN showed a significant overall survival outcome when the survival time was more than 700 days (Fig. 1D). In our collected serum samples, we found that high expression of SRGN was showed a significant overall survival (OS) outcome (Fig. 1J). Multivariate analyses further indicated that elevated SRGN expression is an independent, unfavorable prognostic indicator in HCC patients (Fig. 1K). Taken together, these analyses revealed that high SRGN levels in HCC are significantly correlated with poor patient outcomes.

\section{The proteoglycan SRGN Promotes HCC Cell Migration and Metastasis in an ECM Autocrine Manner}

To confirm the findings described above, quantitative real-time PCR and immunoblotting were performed to quantify the mRNA levels of SRGN among the HCC cell lines, SRGN mRNA and protein expression were differentially expressed among the different HCC cell lines (Fig. 2A). To explore the role of SRGN in HCC cell growth, we transfected SK-Hep-1 cells with shRNA or negative control. Notably, we confirmed that secreted SRGN protein of approximately $300 \mathrm{kDa}$ in molecular weight was significantly decreased in the conditioned medium (CM) (Fig. 2B and 2C). We observed the SRGN suppression was not significant in HCC proliferation ability (Supplementary Fig. 1B). Additionally, we generated cell lines overexpressing SRGN in Hep 3B cells, and SRGN protein expression was validated by qPCR and immunoblotting (Fig. 2B and $2 \mathrm{C}$ ). Overexpression of SRGN in Hep 3B showed a similar result, with no significant difference in the HCC proliferation ability (Supplementary Fig. 1C).

To explore the relationship between SRGN and the metastatic process, we performed wound healing assays to evaluate the migratory ability of SRGN. SRGN knockdown dramatically reduced the migratory ability, and SRGN overexpression increased the migratory ability (Fig. 2D and 2E). To confirm this result, we performed the transwell assay, which showed that SRGN knockdown largely reduced the migratory ability, and SRGN overexpression promoted migration (Fig. 2F, G). These results indicate that SRGN specifically functions by promoting migration in HCC cell lines. However, we found that the knockdown of SRGN expression resulted in the downregulation of the levels of EMT-promoting factors, such as Ncadherin, vimentin, and MMP2, and the upregulation of epithelial markers, such as E-cadherin, in SK-Hep1 cells (Fig. $2 \mathrm{H}$ ). Conversely, SRGN overexpression resulted in the upregulation of $\mathrm{N}$-cadherin, vimentin and MMP2 and downregulation of E-cadherin in Hep 3B cells.

To validate the metastatic function of SRGN in HCC cell lines in vivo, SRGN KD \#2 and control cells were injected into the tail veins of nude mice. After 8 weeks, the mice were euthanized and their lungs were excised and examined. Using hematoxylin and eosin (H\&E) staining, the metastatic nodules in the lung tissue of SRGN KD\#2 mice were observed to be fewer and smaller than those in the control mice (Fig. 2I and $2 \mathrm{~K}$ ). The bodyweight of the KD \#2 group was higher than that of the control group (Fig. 2J). These results strongly suggest that SRGN is closely associated with metastasis in HCC cells.

\section{SRGN is Significantly Associated with Stemness-Like Properties in HCC Cells}


Recent studies have shown that CSCs are highly metastatic and may be quite plastic and associated with EMT. To explore whether SRGN can impact cancer stem cell ability, we used MHCC-97H cells, a suitable cell line model to perform the self-renewal assay, and the knockdown efficiency is shown in Fig. $3 \mathrm{~A}$ and 3B. To examine the effect of SRGN on maintaining cancer stem cell characteristics, we performed a sphere culture assay, and SRGN knockdown reduced the number of spheres generated by MHCC-97H cells (Fig. 3C and 3D), indicating the critical role of SRGN in the self-renewal capacity of the cells. CSCs have the capacity for self-renewal and differentiation potential, and previous studies [26] have demonstrated that the side population (SP) cells, identified by their ability to pump out a fluorescent dye (Hoechst 33342), have certain characteristics of CSCs, the SP phenotype can be a CSC marker for HCC. Herein, we found that the side population in MHCC-97H cells with SRGN knockdown was reduced compared with control cells (Fig. 3E and 3F). Overexpression of Hep 3B cells with SRGN showed that ABCG2, Bmi-1, and Nanog were largely increased. Additionally, we suppressed SRGN in MHCC-97H cells and tested the three different CSC markers. ABCG2, Bmi-1, and Nanog were reduced at the protein level, indicating a promoted HCC self-renewal capacity (Fig. 3G). More importantly, the SP sorting assay revealed that SRGN was upregulated in the side population, indicating more CSC-like ability in HCC (Fig. $3 \mathrm{H}$ ). These observations suggest that SRGN is closely associated with the CSC-like properties of HCC cells.

\section{SRGN with its Receptor CD44 Regulates the Nuclear Localization of the Hippo Pathway Effector YAP}

Our previous study[14] showed that SRGN induces CD44 expression to potentiate its self-renewal capacity by activating the MAPK pathway in NPC, and CD44 was a receptor of the ECM ligand SRGN. Therefore, we examined the expression of CD44 and SRGN in HCC. The relative expression level of CD44 was increased and decreased after SRGN suppression and overexpression, respectively (Fig. 4A). Notably, the TCGA database showed a positive correlation between CD44 and SRGN in primary HCC tissues (Fig. 4B). These results indicate that SRGN expression is positively correlated with CD44 expression in both HCC cell lines and tissues.

The Hippo/YAP pathway has been delineated and shown to play an important role in regulating tissue proliferation and apoptosis rates. It is highly expressed in stem cells and associated with the CSC capacity. Based on the result that SRGN can significantly promote self-renewal ability, we hypothesized that promotion of CSC ability by SRGN might be associated with the Hippo/YAP pathway.

To further explore whether SRGN plays a crucial role in the YAP pathway, we found that SRGN was positively correlated with YAP expression in HCC cell lines (Fig. 4C). In mammals, MST1 is a core kinase cascade in the YAP pathway that can phosphorylate the kinase LATS1, and then the LATS1 complex phosphorylates and represses the transcriptional coactivator YAP. We also found that the phosphorylation of YAP was decreased and the phosphorylation of MST1 and LATS1 was increased, as detected by immunoblotting (Fig. 4D). Thus, SRGN suppresses the YAP pathway via inhibiting YAP phosphorylation. When SRGN is overexpressed, YAP expression was increased, particularly in the nucleus 
(Fig. 4E). To confirm this result, we performed the confocal immunofluorescence assay to examine the nuclear and cytosolic/membrane fractions and found that SK-Hep-1 knockdown cells with less YAP translocated to the nucleus (Fig. 4F), whereas more YAP translocated to the nucleus in SRGNoverexpressed Hep 3B cells (Fig. 4G). The analysis of SRGN knockdown and overexpression cells revealed that SRGN affects YAP expression in the nucleus significantly. To elucidate the function of SRGN in HCC progression, we generated the DEN/CCL4 induced-HCC mouse model to evaluate the correlation of SRGN and YAP in the process of HCC mouse model formation. Confocal immunofluorescence assay showed that HCC tumors formed in the mouse model, increased YAP and SRGN expression was observed in liver tumor tissues, and YAP was highly expressed in the nucleus (Fig. 4H). Thus, SRGN regulates YAP expressionand and the nuclear localization of YAP in HCC cells and mouse models.

\section{CRISPLD2 is a SRGN-Mediated HIPPO/YAP Pathway Downstream Effector Gene and Potentiates HCC Cell Motility Ability}

To explore the downstream targets of SRGN that promotes HCC metastasis, we performed whole-genome expression profiling in SK-Hep-1 cells and Hep 3B cells. Given that SRGN promotes cancer metastasis, we focused on the SRGN-regulated genes implicated in these processes from microarray analysis (Fig. 5A). Notably, CRISPLD2, also named LGL1, overlapped in the knockdown and overexpression groups, and the increased expression of CRISPLD2 by SRGN overexpression was validated by qPCR and immunoblotting at the mRNA and protein levels, respectively (Fig. 5C,D; Supplementary Fig. 1D, E), and SRGN was highly correlated with CRISPLD2 in HCC cell lines and TCGA database (Fig. 5B, C). Using siRNAs to suppress CRISPLD2, we found that vimentin expression was also decreased (Fig. 5G), a finding that was similar to the result after the knockdown of SRGN expression. Cell growth assays showed that the knockdown of CRISPLD2 can significantly inhibit cell proliferation (Supplementary Fig. 1F). The Transwell assay showed that CRISPLD2 knockdown can inhibit cell migration ability (Fig. 5E). Overexpression of SRGN in Hep 3B cells was validated by qPCR and immunoblotting (Fig. 5F), and the migration assay showed that SRGN promotes metastasis in vitro (Fig. 5E).

The YAP-TEAD1 protein-protein interaction (PPI) mediates the oncogenic function of YAP, and inhibitors of this PPI have potential use in the treatment of YAP-involved cancers [27]. Peptide 17 is a promising inhibitor that can efficiently disrupt the interaction between YAP and TEAD1. Peptide 17 induction can inhibit the SRGN overexpression effects in increasing CRISPLD2 expression, while with no effects observed in the vector group (Fig. $5 \mathrm{H}$ ). Immunoblotting showed that the CRISPLD2 protein level decreased after peptide 17 induction in the SRGN overexpression group compared with that in the vector group (Fig. 5I). Thus, peptide 17 can suppress CRISPLD2 expression by inhibiting SRGN expression.

To further confirm that ECM proteoglycan ligand SRGN induces the expression of its target gene CRISPLD2, CRISPLD2 promoter luciferase reporter assay revealed increased activity with SRGN overexpression. However, after peptide 17 induction, the regulation of CRISPLD2 activity by SRGN was 
suppressed (Fig. 5J, K). The transwell assay showed that suppressed SRGN could decrease the migration ability. Additionally, CRISPLD2 overexpression after SRGN suppression increased the migration ability to some extent (Fig. 5L, M, N; Supplementary Fig. 1G).

Next, we determined whether SRGN promotes the migration ability in the YAP pathway. We treated the cells with verteporfin (VP), which disrupts the formation of the YAP-TEAD1 complex, significantly reducing preneoplastic foci and oval cell proliferation. Treatment with different doses of VP decreased the migration ability of SRGN-overexpressing cells. CRISPLD2 suppression reversed the SRGN-promoting migration ability (Fig. 50). Collectively, these results strongly suggest that the promotion of HCC metastasis by upregulating SRGN depends on the up-regulation of CRISPLD2. CRISPLD2 is a SRGNmediated HIPPO/YAP pathway downstream effector gene and potentiates HCC cell motility ability.

\section{CRISPLD2 Is a Novel YAP-TEAD1 Target Gene Dependent on SRGN}

To further confirm that SRGN mediates the downstream target gene CRISPLD2 and YAP pathway, we transiently transfected MHCC-97H cells with YAP. As expected, the CRISPLD2 promoter luciferase reporter assay revealed increased activity of the CRISPLD2 promoter with YAP overexpression (Fig. 6A). When treated with verteporfin, the CRISPLD2 promoter was suppressed (Fig. 6B), suggesting that the activity of the CRISPLD2 promoter was directly regulated by the YAP-activating specific signaling pathway.

We used the Jasper database (http://jaspar.binf.ku.dk/) to predict that the co-transcription factor YAPTEAD1 has two potential binding sites in the promoter region of CRISPLD2. The luciferase assay proved that the deletion of site 1 reduced the activity of luciferase, but the deletion of site 2 showed no significant change in the activity of luciferase (Fig. 6C). Thus, the co-transcription factor YAP-TEAD1 accelerates CRISPLD2 transcription through binding site 1 (TGGATTCCTGGG).

To confirm the mechanism study, we used chromatin immunoprecipitation (ChIP). Based on the CRISPLD2 promoter region, we designed 5 pairs of PCR primers (Fig. 6D). The CRISPLD2 promoter sequence was detected by qPCR and normalized to the input level (Fig. 6E and 6F). The data revealed that YAP-TEAD1 directly binds to the CRISPLD2 promoter and activates CRISPLD2 transcription, and two binding sites in CRISPLD2 promoter are regulated by YAP-TEAD1. However, YAP/TEAD1 target genes (BIRC5, AREG, CCND1, CTGF and MYC) were not activated by SRGN-mediated HIPPO/YAP signals in HCC cells (Supplementary Fig. $1 \mathrm{H}$ ), indicating that CRISPLD2 is a novel selective YAP-TEAD1 target gene in the SRGN-mediated HIPPO/YAP signaling pathway.

Cotreatment with the YAP-TEAD1 inhibitor and sorafenib inhibits tumor growth in vivo and in vitro

We further investigated whether SRGN directly promotes the sorafenib resistance by performing flow cytometry and measuring the percentage of apoptosis 2 days after sorafenib induction. In the KD\#1 and $K D \# 2$ groups, as the drug concentration increased, the apoptosis rate was increased less than that in the 
control group (Fig. 7A, C). Additionally, sorafenib largely induced apoptosis in SRGN overexpression cells than vector cells in a dose-dependent manner (Fig. 7B, D).

To further explore the signaling pathways induced by SRGN under sorafenib treatment, we first checked the total phosphorylated ERK, P-ERK, YAP and P-YAP in SK-Hep-1 and Hep 3B cell lines. Hep-3B cells treated with sorafenib after overexpressing SRGN, the hallmarks of apoptosis such as the induction of caspase-3 cleavage occurred in a dose-dependent manner (Fig. 7E, F), and the effect of sorafenib on the YAP signaling pathway in HCC cells indicated that targeting SRGN can inhibit cell apoptosis in the YAP pathway. Sorafenib could abrogate the stimulatory effects of SRGN on YAP, P-YAP, ERK, and P-ERK. Moreover, we found that overexpressing cells had higher cleaved caspase-3 levels than vector cells, but they had lower caspase-3 levels than the vector cells in a sorafenib dose-dependent manner.

The cell viability assay showed that VP could reverse the suppression of sorafenib-induced cell apoptosis by SRGN-overexpressing cells (Fig. 7G and $7 \mathrm{H}$ ). Additionally, colony formation showed that VP and sorafenib combination treatment could largely decrease the cell colony formation ability (Fig. $7 \mathrm{I}$ and $7 \mathrm{~J}$ ). The confocal immunofluorescence assay showed that YAP was highly expressed in nuclei of the side population, revealing that YAP likely translocates the nucleus compared with the main population (Fig. 7K).

The SP test showed that verteporfin could significantly reduce the side population from $14.67-0.29 \%$, while sorafenib can reduce the cells of the main population from $10 \times 10^{6}$ to $6.87 \times 10^{6}$ but not those in the verteporfin group (Fig. 7L, M). Sorafenib combined with verteporfin greatly reduced the main and side populations of MHCC $97 \mathrm{H}$ cells.

Next, an SK-Hep-1 xenograft model was established in nude mice to investigate the in vivo effects of the above findings. The results showed that the tumor volume was much smaller in the combination therapy groups than in the single-drug (sorafenib or verteporfin) groups (Fig. 70, P). Additionally, the results were further evaluated by IHC staining of c-caspase 3 and YAP in ex vivo tumor samples (Fig. 7Q). Taken together, sorafenib combined with Verteporfin can reverse the SRGN-induced sorafenib resistance through targeting YAP-activated CSCs.

\section{Discussion}

Metastasis is the major cause of treatment failure in patients with HCC. Thus, the prevention, prediction, and inhibition of metastasis are crucial to improving treatment outcomes. Currently, serum tumor markers are commonly used as a diagnostic method for HCC [28]. SRGN is an extracellular matrix (ECM) component, and its level in liver cancer patients without metastasis is higher than that in healthy volunteers and patients without metastasis. Furthermore, the expression of SRGN in serum is positively correlated with AFP, which is another significant biomarker for HCC diagnosis in patient serum. Accumulating studies have shown that the ECM contributes to the maintenance of CSC self-renewal in an 
autocrine manner in many cancers [29]. Moreover, CSCs were suggested to drive tumor initiation and progression and are closely associated with sorafenib resistance, recurrence, and metastasis [26].

In the present study, we reported the high expression of SRGN in primary tissues and serum with metastasis in contrast to low expression of SRGN in primary tissues and serum without metastasis. Moreover, elevated SRGN expression in primary HCC was found to be an independent, unfavorable prognostic factor for patients. These studies strongly indicate that SRGN plays a critical role in HCC progression and is an independent marker of metastases in HCC.

SRGN is a proteoglycan that is the earliest reported factor to be secreted by hematopoietic cells [30]. As a typical ECM component, SRGN participates in packaging secretory granules and regulates the storage or release of enzymes, serotonin and histamine in hematopoietic cells [31]. SRGN is upregulated in nasopharyngeal carcinoma and breast cancer [14], and it promotes tumor growth, migration, invasion, and angiogenesis. However, to date, no study has been conducted to elucidate the underlying mechanism of SRGN-mediated activation of the signaling pathway in HCC. In this study, we used the TCGA database to analyze whether SRGN is upregulated in primary tumor with metastasis (Fig. 1B). Functionally, we found that the knockdown of SRGN could induce HCC cell migration in vitro, as well as metastasis in vivo, while the overexpression of SRGN promoted migration (Fig. 2F and 2J). Additionally, SRGN downregulation enhances the sensitivity of SK-Hep-1 cells to sorafenib therapy (Fig. 7A and 7B), suggesting that targeting multiple factors is a promising strategy for the complete suppression of HCC metastasis.

The cellular surface adherent CD44 molecule is a common CSC marker in several tumors and a $\beta$-cateninmediated transcriptional target [32]. Previous studies and other researchers have demonstrated that CD44 serves as an SRGN receptor is a potential CSC marker [33]. Consistently, in our study, a positive correlation was observed between the expression of SRGN and CRISPLD2 using clinical HCC samples. TCGA database revealed that CD44 expression is strongly correlated with SRGN expression (Fig. 4B). Importantly, we found that SRGN induces ABCG2, Bmi1, and Nanog expression to potentiate the cells' self-renewal capacity and SP percentage (Fig. 3G). The ECM component SRGN, as a CD44 ligand, plays a crucial role in maintaining $\mathrm{HCC}$ cell stemness and contributes to $\mathrm{HCC}$ cell sorafenib resistance.

To explore the downstream targets of SRGN that promotes HCC metastasis, we performed whole-genome expression profiling using stable SRGN shRNAs in SK-Hep-1 cell lines and SRGN overexpression in Hep 3B cells. Given that SRGN promotes cancer metastasis, we focused on SRGN-regulated genes implicated in these processes from this microarray analysis.

Cysteine-rich secretory protein LCCL domain-containing 2 is a cysteine-rich secretory protein that, in humans, is encoded by the CRISPLD2 gene [34]. Diseases associated with CRISPLD2 include orofacial cleft 15 and cleft lip. Among its related pathways is the innate immune system. CRISPLD2 is significantly associated with craniofacial morphogenesis [35] as well as alveolar development and branching morphogenesis [36].Notably, CRISPLD2 expression overlapped in both the knockdown and overexpression groups. CRISPLD2, also called late gestation lung 1 (Lgl1), has been implicated in the 
development of the rat lung, mouse kidney, and human fetal lung fibroblast cells [37]. CRISPLD2 was also shown to be associated with the occurrence of non-syndromic left cleft lip or without cleft disease [38]. Consistently, in this study, a positive correlation was observed between the expression of SRGN and CRISPLD2 using clinical HCC samples. For the first time, we found that SRGN expression is closely associated with CRISPLD2 expression by activating the YAP pathway. The YAP pathway is crucial in animal organ size control by regulating tissue proliferation and apoptosis rates as a response to developmental cues, cell contact, and density. YAP expression and nuclear localization strongly correlate with poor patient outcomes and the progression of several tumor types. Both CRISPLD2 and YAP pathways are defined as genes related to embryonic development, and the YAP/CRISPLD2 developmental axis was reactivated selectively in SRGN-mediated HCC cells, instead of other target genes, such as BIRC5, AREG, CCND1, CTGF and MYC.

Verteporfin is a promising inhibitor that can efficiently disrupt the interaction between YAP and TEAD1[39]. Verteporfin induction could reverse CRISPLD2 expression via overexpressing SRGN. CRISPLD2 overexpression could also reverse the migration phenotype after SRGN knockdown. We presume that SRGN overexpression is a candidate factor responsible for the activation of YAP signaling in HCC.

As a targeted drug, sorafenib is the first systemic drug approved for patients with advanced HCC to prevent $\mathrm{HCC}$ recurrence. Therefore, it is currently used as the standard treatment for patients with advanced HCC [40]. The high expression of SRGN in liver cancer may endow it with resistance to sorafenib, making it a major candidate drug for combined use with other safe cancer treatment drugs. Additionally, the SRGN-mediated Hippo/YAP signaling pathway is activated, and the co-occurrence of changes suggests the opportunity for combination therapy. Interestingly, the combination therapy of sorafenib and verteporfin showed significant anti-tumor effects both in vivo and in vitro, particularly in SRGN high expression cells. Sorafenib plus verteporfin greatly reduces the bulk tumor and tumor stem cells. These findings may provide promising new guidance for personalized precision therapy in SRGN positive HCC treatment and are expected to have potential applications in other cancers.

In summary, our findings are the first to elucidate the crucial roles of SRGN in promoting HCC metastasis and predicting an unfavorable patient prognosis. In particular, SRGN is positively correlated with CRISPLD2 and activated YAP signaling. Sorafenib plus verteporfin can reverse SRGN-induced sorafenib resistance through targeting YAP-activated CSCs. Considering the findings observed in the present study, a proposed model illustrating the oncogenic effect of SRGN in the pathogenesis of HCC is provided in Fig. 7R.

\section{Conclusions}

Autocrine ECM serglycin plays a crucial role in the process of stemness maintenance, tumorigenesis, and metastasis via selective reactivation of the novel YAP/CRISPLD2 developmental axis promotes 
metastasis and sorafenib resistance in Hepatocellular carcinoma, Verteporfin (YAP inhibitor) combined with sorafenib reversed serglycin-mediated cellular insensitivity to sorafenib treatment;

\section{Abbreviations}

$\mathrm{HCC}$

Hepatocellular carcinoma

ECM

extracellular matrix

EMT

epithelial-to-mesenchymal transition

YAP1

yes-associated protein 1

CRISPLD2

Cysteine-rich secretory protein LCCL domain-containing 2

DEN

diethylmitrosamine

SRGN

serglycin

CSC

cancer stem cell

TCGA

The Cancer Genome Atlas

\section{Declarations}

\section{Ethics approval and consent to participate}

All animal experiments were approved by the Institutional Animal Care and Use Committee of the Sun Yat-Sen University Cancer Center. All human serum samples were obtained with patient consent and the approval of the Institutional Clinical Ethics Review Board at Sun Yat-Sen University Cancer Center.

\section{Consent for publication}

Not applicable.

\section{Availability of data and materials}

Not applicable. 


\section{Competing interests:}

The authors declare that they have no competing interests.

\section{Funding}

This work was supported by grants from the National Natural Science Foundation of China (No. $82073220 \rrbracket$ No. 81972785 , No. 81773162 , No.81572901, No. 81872384 , No. 81672872 , No. 81873284 and No.81673903), the Provincial Natural Science Foundation of Guangdong, China (No. 2017A030313866) .

\section{Authors' contributions}

The authors are grateful to Shijun Zhang, Chaonan Qian and Bijun Huang for helpful suggestions. Author Contributions: Hao Hu, Liang $\mathrm{Xu}$, and Li-sheng Zheng designed the experiments and interpreted the results. Hao Hu, Liang Xu, Tie-Jun Huang, Yan Mei, Li-sheng Zheng, Li-xia Peng, performed the experiments. Fei-fei Luo, Jing Wang, Zhan-fei Zhang and Hao Hu analyzed the clinical data Dongfang Meng improved the project design and interpreted the results. Hao Hu and Liang Xu wrote the manuscript, which was further refined by all authors. The authors confirm that there are no conflicts of interest.

\section{Acknowledgments}

Not applicable.

\section{References}

1. Forner A, Llovet JM, Bruix J. Hepatocellular carcinoma. Lancet. 2012;379(9822):1245-55.

2. Hu H, Xu L, Luo SJ, Xiang T, Chen Y, Cao ZR, Zhang YJ, Mo Z, Wang Y, Meng DF, et al. Retinal dehydrogenase 5 (RHD5) attenuates metastasis via regulating HIPPO/YAP signaling pathway in Hepatocellular Carcinoma. Int J Med Sci. 2020;17(13):1897-908.

3. Lambert AW, Pattabiraman DR, Weinberg RA. Emerging Biological Principles of Metastasis. Cell. 2017;168(4):670-91.

4. Emma MR, lovanna JL, Bachvarov D, Puleio R, Loria GR, Augello G, Candido S, Libra M, Gulino A, Cancila V, et al. NUPR1, a new target in liver cancer: implication in controlling cell growth, migration, invasion and sorafenib resistance. Cell Death Dis. 2016;7(6):e2269.

5. Pejler G, Abrink M, Wernersson S. Serglycin proteoglycan: regulating the storage and activities of hematopoietic proteases. Biofactors. 2009;35(1):61-8.

6. Kolset SO, Tveit H. Serglycin-structure and biology. Cell Mol Life Sci. 2008;65(7-8):1073-85. 
7. Schick BP, Ho H-CK, Brodbeck KC, Wrigley CW, Klimas J. Serglycin proteoglycan expression and synthesis in embryonic stem cells. Biochim Biophys Acta. 2003;1593(2-3):259-67.

8. Biederbick A, Licht A, Kleene R. Serglycin proteoglycan is sorted into zymogen granules of rat pancreatic acinar cells. Eur J Cell Biol. 2003;82(1):19-29.

9. Theocharis AD, Seidel C, Borset M, Dobra K, Baykov V, Labropoulou V, Kanakis I, Dalas E, Karamanos NK, Sundan A, et al. Serglycin constitutively secreted by myeloma plasma cells is a potent inhibitor of bone mineralization in vitro. J Biol Chem. 2006;281(46):35116-28.

10. Purushothaman A, Toole BP. Serglycin proteoglycan is required for multiple myeloma cell adhesion, in vivo growth, and vascularization. J Biol Chem. 2014;289(9):5499-509.

11. Li XJ, Ong CK, Cao Y, Xiang YQ, Shao JY, Ooi A, Peng LX, Lu WH, Zhang Z, Petillo D, et al. Serglycin is a theranostic target in nasopharyngeal carcinoma that promotes metastasis. Cancer research. 2011;71(8):3162-72.

12. Xu Y, Xu J, Yang Y, Zhu L, Li X, Zhao W. SRGN Promotes Colorectal Cancer Metastasis as a Critical Downstream Target of HIF-1a. Cell Physiol Biochem. 2018;48(6):2429-40.

13. Zhang Z, Deng Y, Zheng G, Jia X, Xiong Y, Luo K, Qiu Q, Qiu N, Yin J, Lu M, et al. SRGN-TGFß2 regulatory loop confers invasion and metastasis in triple-negative breast cancer. Oncogenesis. 2017;6(7):e360.

14. Chu Q, Huang H, Huang T, Cao L, Peng L, Shi S, Zheng L, Xu L, Zhang S, Huang J, et al. Extracellular serglycin upregulates the $C D 44$ receptor in an autocrine manner to maintain self-renewal in nasopharyngeal carcinoma cells by reciprocally activating the MAPK/ $\beta$-catenin axis. Cell Death Dis. 2016;7(11):e2456.

15. Ma Y, Yang Y, Wang F, Wei Q, Qin H. Hippo-YAP signaling pathway: A new paradigm for cancer therapy. International journal of cancer. 2015;137(10):2275-86.

16. Fernandez LA, Northcott PA, Dalton J, Fraga C, Ellison D, Angers S, Taylor MD, Kenney AM. YAP1 is amplified and up-regulated in hedgehog-associated medulloblastomas and mediates Sonic hedgehog-driven neural precursor proliferation. Genes Dev. 2009;23(23):2729-41.

17. Zhao B, Wei X, Li W, Udan RS, Yang Q, Kim J, Xie J, Ikenoue T, Yu J, Li L, et al. Inactivation of YAP oncoprotein by the Hippo pathway is involved in cell contact inhibition and tissue growth control. Genes Dev. 2007;21(21):2747-61.

18. Steinhardt AA, Gayyed MF, Klein AP, Dong J, Maitra A, Pan D, Montgomery EA, Anders RA. Expression of Yes-associated protein in common solid tumors. Hum Pathol. 2008;39(11):1582-9.

19. Hall CA, Wang R, Miao J, Oliva E, Shen X, Wheeler T, Hilsenbeck SG, Orsulic S, Goode S. Hippo pathway effector Yap is an ovarian cancer oncogene. Cancer research. 2010;70(21):8517-25.

20. Ramalho-Santos M, Yoon S, Matsuzaki Y, Mulligan RC, Melton DA. "Stemness": transcriptional profiling of embryonic and adult stem cells. Science. 2002;298(5593):597-600.

21. Camargo FD, Gokhale S, Johnnidis JB, Fu D, Bell GW, Jaenisch R, Brummelkamp TR. YAP1 increases organ size and expands undifferentiated progenitor cells. Current biology: CB. 2007;17(23):2054-60. 
22. Liu L, Cao Y, Chen C, Zhang X, McNabola A, Wilkie D, Wilhelm S, Lynch M, Carter C: Sorafenib blocks the RAF/MEK/ERK pathway, inhibits tumor angiogenesis, and induces tumor cell apoptosis in hepatocellular carcinoma model PLC/PRF/5. Cancer research 2006, 66(24):11851-11858.

23. Anido J, Sáez-Borderías A, Gonzàlez-Juncà A, Rodón L, Folch G, Carmona MA, Prieto-Sánchez RM, Barba I, Martínez-Sáez E, Prudkin L, et al. TGF- $\beta$ Receptor Inhibitors Target the CD44(high)/ld1(high) Glioma-Initiating Cell Population in Human Glioblastoma. Cancer Cell. 2010;18(6):655-68.

24. Suvà ML, Rheinbay E, Gillespie SM, Patel AP, Wakimoto $H$, Rabkin SD, Riggi N, Chi AS, Cahill DP, Nahed BV, et al. Reconstructing and reprogramming the tumor-propagating potential of glioblastoma stem-like cells. Cell. 2014;157(3):580-94.

25. S X, Z F, M Z, Y W, Y S, H X, X L, K H, J C, R Z et al: hSSB1 binds and protects p21 from ubiquitinmediated degradation and positively correlates with p21 in human hepatocellular carcinomas. Oncogene 2011, 30(19):2219-2229.

26. Xu L, Huang TJ, Hu H, Wang MY, Shi SM, Yang Q, Lin F, Qiang YY, Mei Y, Lang YH, et al. The developmental transcription factor IRF6 attenuates ABCG2 gene expression and distinctively reverses stemness phenotype in nasopharyngeal carcinoma. Cancer letters. 2018;431:230-43.

27. Zhang Z, Lin Z, Zhou Z, Shen HC, Yan SF, Mayweg AV, Xu Z, Qin N, Wong JC, Zhang Z, et al. StructureBased Design and Synthesis of Potent Cyclic Peptides Inhibiting the YAP-TEAD Protein-Protein Interaction. ACS Med Chem Lett. 2014;5(9):993-8.

28. Hu H, Xu L, Chen Y, Luo SJ, Wu YZ, Xu SH, Liu MT, Lin F, Mei Y, Yang Q, et al. The Upregulation of Trophinin-Associated Protein (TROAP) Predicts a Poor Prognosis in Hepatocellular Carcinoma. J Cancer. 2019;10(4):957-67.

29. Oskarsson T, Acharyya S, Zhang XH, Vanharanta S, Tavazoie SF, Morris PG, Downey RJ, ManovaTodorova K, Brogi E, Massagué J. Breast cancer cells produce tenascin C as a metastatic niche component to colonize the lungs. Nature medicine. 2011;17(7):867-74.

30. Schick BP, Ho HC, Brodbeck KC, Wrigley CW, Klimas J. Serglycin proteoglycan expression and synthesis in embryonic stem cells. Biochim Biophys Acta. 2003;1593(2-3):259-67.

31. Keith Ho HC, McGrath KE, Brodbeck KC, Palis J, Schick BP. Serglycin proteoglycan synthesis in the murine uterine decidua and early embryo. Biol Reprod. 2001;64(6):1667-76.

32. Zhu X, Morales FC, Agarwal NK, Dogruluk T, Gagea M, Georgescu M-M. Moesin Is a Glioma Progression Marker That Induces Proliferation and Wnt/ $\beta$-Catenin Pathway Activation via Interaction with CD44. Cancer research. 2013;73(3):1142-55.

33. Yang CF, Peng LX, Huang TJ, Yang GD, Chu QQ, Liang YY, Cao X, Xie P, Zheng LS, Huang HB, et al. Cancer stem-like cell characteristics induced by EB virus-encoded LMP1 contribute to radioresistance in nasopharyngeal carcinoma by suppressing the p53-mediated apoptosis pathway. Cancer letters. 2014;344(2):260-71.

34. Wiemann S, Weil B, Wellenreuther R, Gassenhuber J, Glassl S, Ansorge W, Böcher M, Blöcker H, Bauersachs $\mathrm{S}, \mathrm{Blum} \mathrm{H}$, et al. Toward a catalog of human genes and proteins: sequencing and analysis of 500 novel complete protein coding human cDNAs. Genome Res. 2001;11(3):422-35. 
35. Yuan Q, Chiquet BT, Devault L, Warman ML, Nakamura Y, Swindell EC, Hecht JT. Craniofacial abnormalities result from knock down of nonsyndromic clefting gene, crispld2, in zebrafish. Genesis. 2012;50(12):871-81.

36. Oyewumi L, Kaplan F, Gagnon S, Sweezey NB. Antisense oligodeoxynucleotides decrease LGL1 mRNA and protein levels and inhibit branching morphogenesis in fetal rat lung. Am J Respir Cell Mol Biol. 2003;28(2):232-40.

37. Zhang H, Kho AT, Wu Q, Halayko AJ, Limbert Rempel K, Chase RP, Sweezey NB, Weiss ST, Kaplan F. CRISPLD2 (LGL1) inhibits proinflammatory mediators in human fetal, adult, and COPD lung fibroblasts and epithelial cells. Physiol Rep 2016, 4(17).

38. Chiquet BT, Lidral AC, Stal S, Mulliken JB, Moreno LM, Arcos-Burgos M, Valencia-Ramirez C, Blanton SH, Hecht JT. CRISPLD2: a novel NSCLP candidate gene. Hum Mol Genet. 2007;16(18):2241-8.

39. Wu D, Luo L, Yang Z, Chen Y, Quan Y, Min Z. Targeting Human Hippo TEAD Binding Interface with YAP/TAZ-Derived, Flexibility-Reduced Peptides in Gastric Cancer. International Journal of Peptide Research and Therapeutics 2020.

40. Sun X, Niu X, Chen R, He W, Chen D, Kang R, Tang D. Metallothionein-1G facilitates sorafenib resistance through inhibition of ferroptosis. Hepatology. 2016;64(2):488-500.

\section{Figures}




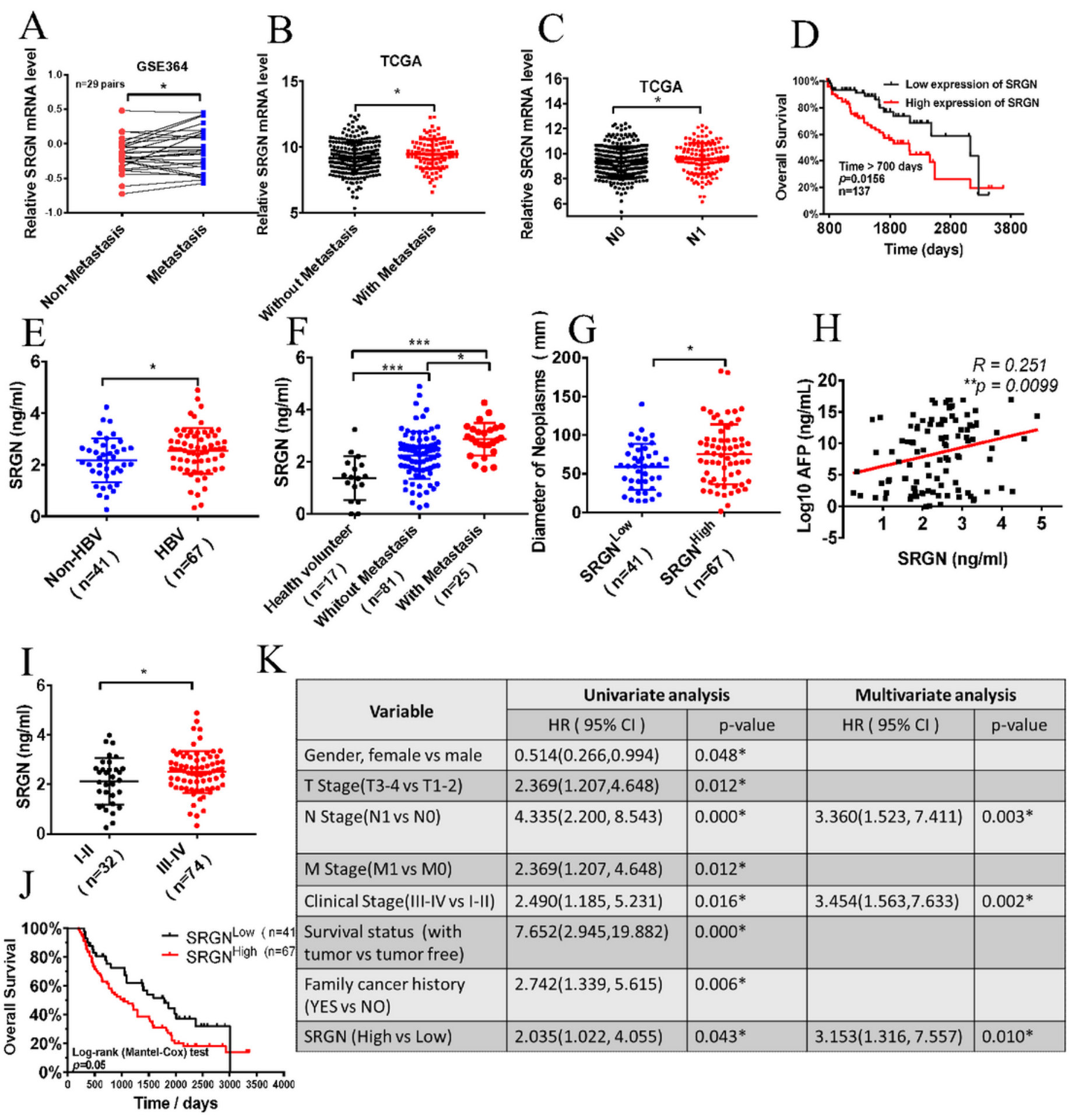

\section{Figure 1}

SRGN is an Independent Prognostic Factor and Potential Serum Biomarker in HCC Patients In the GSE364 cohort (A) and TCGA database (B), the expression of SRGN in metastatic tissues was significantly higher than that in non-metastatic tissues. In the TCGA database, the SRGN mRNA levels were positively correlated with lymph node metastasis (C) and overall survival outcome (D). The expression of SRGN in the patient's serum was positively correlated with HBV infection (E), tumor metastasis (F), tumor size (G), 
AFP $(H)$, clinical stage $(I)$, and overall survival $(J)$. (K) SRGN expression is an independent, poor prognostic indicator for liver cancer patients. ${ }^{*} p<0.05,{ }^{*} p<0.01$.

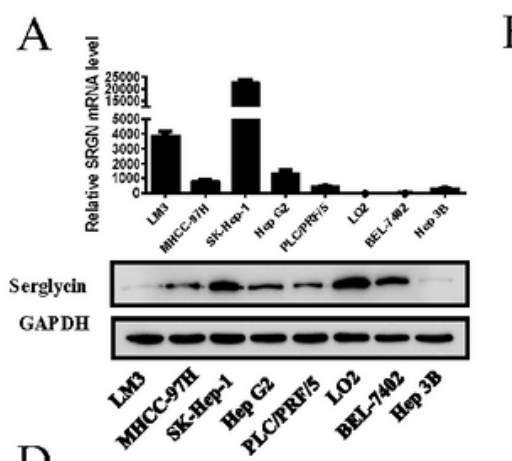

$\mathrm{D}$

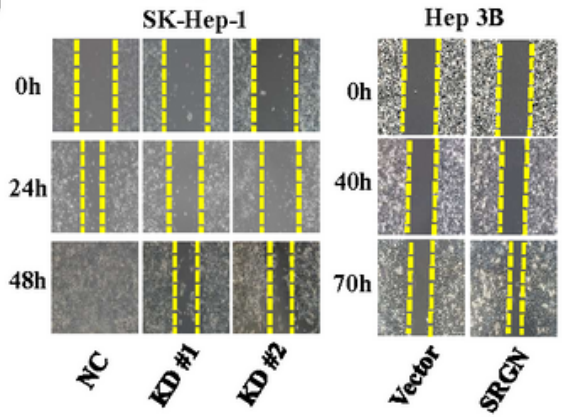

B

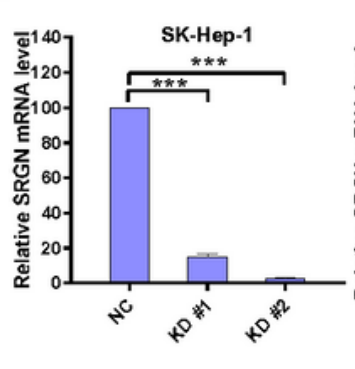

$\mathrm{E}$
C

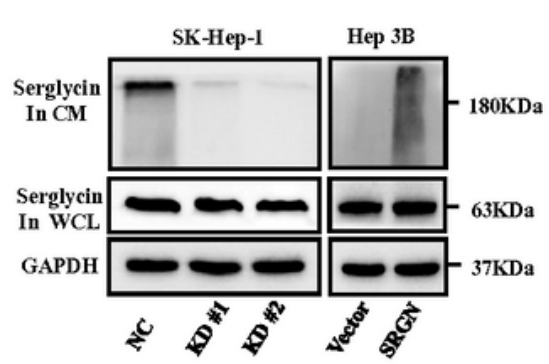

E $\quad$ NC
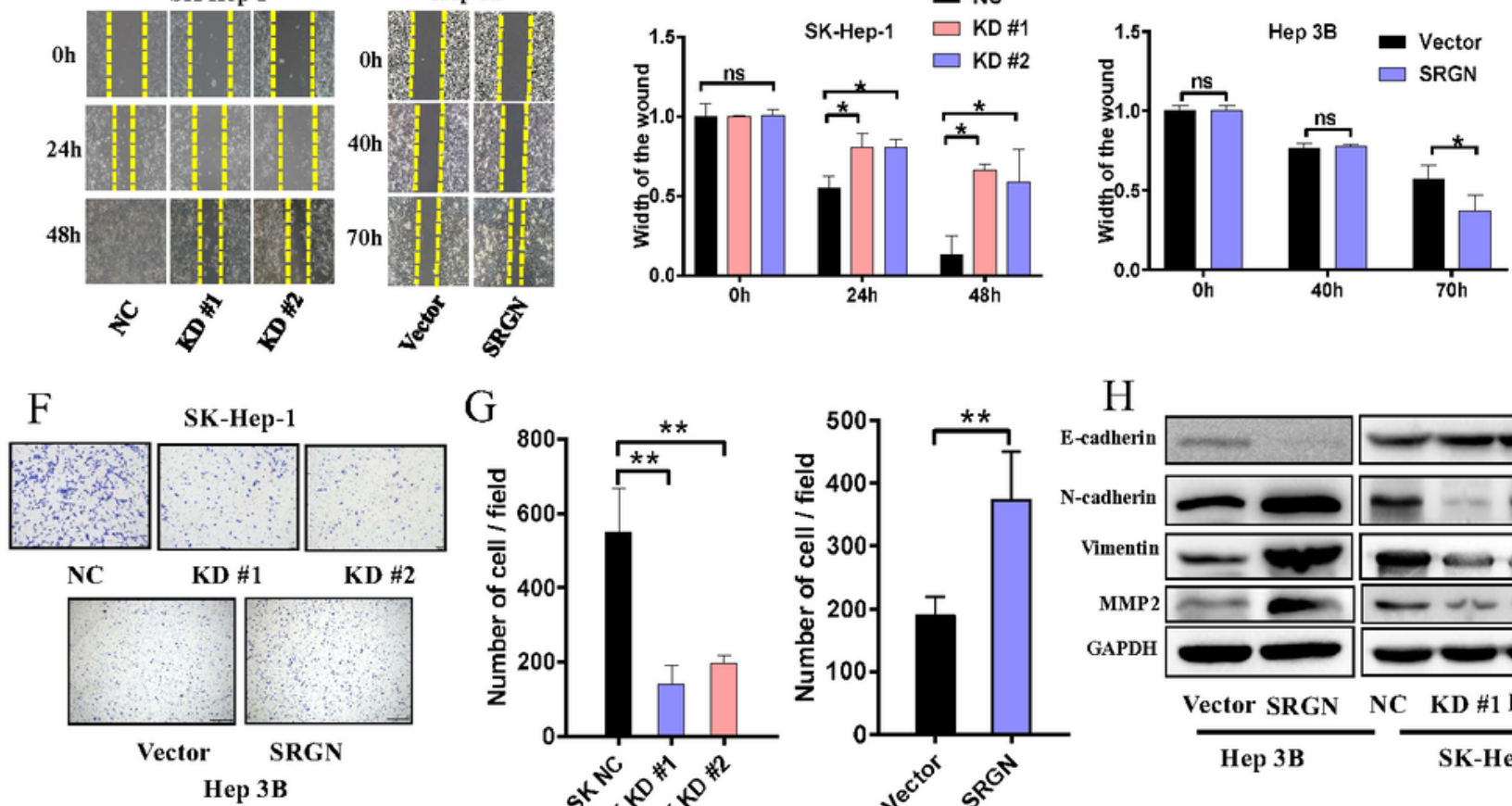

$\mathrm{G}$
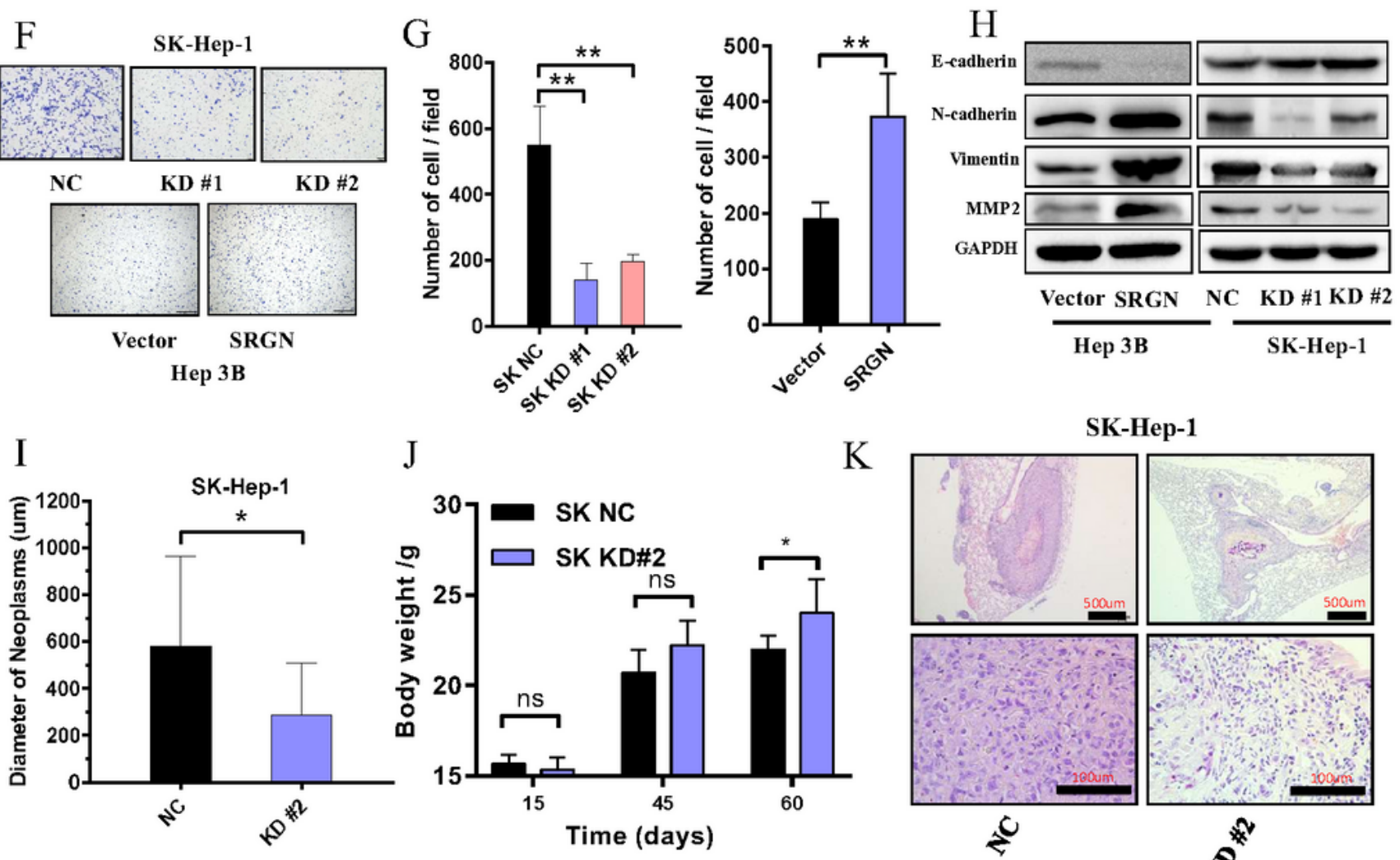

SK-Hep-1

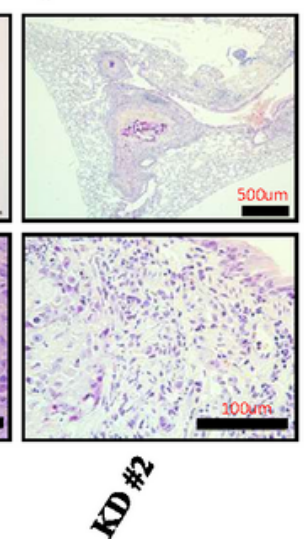

Figure 2

Proteoglycan SRGN Promotes HCC Cell Migration and Metastasis in an ECM Autocrine Manner (A) Differential expression of SRGN mRNA and protein in HCC cells by qPCR and immunoblotting. (B) The knockdown and overexpression of SRGN in HCC cells were monitored by GPCR and immunoblotting. The 
gain and loss of SRGN expression greatly affected the migration ability of cells in the wound healing assay (D, E) and Transwell assay $(F, G)$ in HCC cells. $(H)$ Transient suppression and overexpression of SRGN in HCC cells and whole cell lysates, followed by immunoblotting for E-cadherin, N-cadherin, Vimentin, and MMP2 at $72 \mathrm{~h}$ after transfection. Inhibition of SRGN reduced the transfer efficiency of SKHep-1 cells (I) in vivo and increased the bodyweight of nude mice $(\mathrm{J})$. (K) Histological image of lung metastasis in nude mice after tail vein injection of SK-Hep-1 cells. ${ }^{*} p<0.05,{ }^{* *} p<0.01$.

A

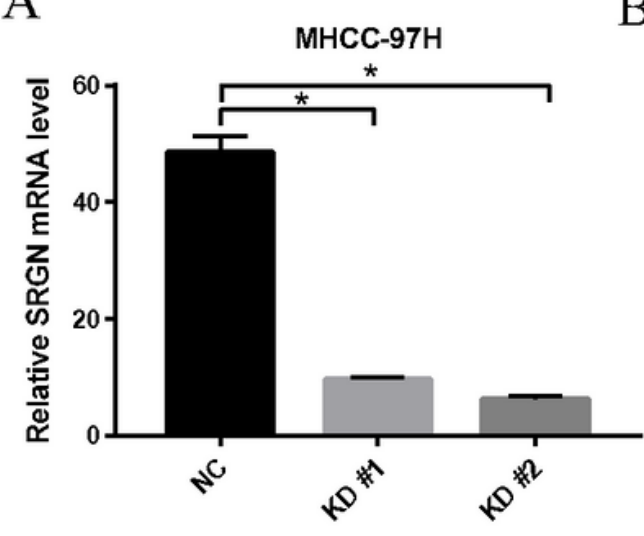

$\mathrm{D}$
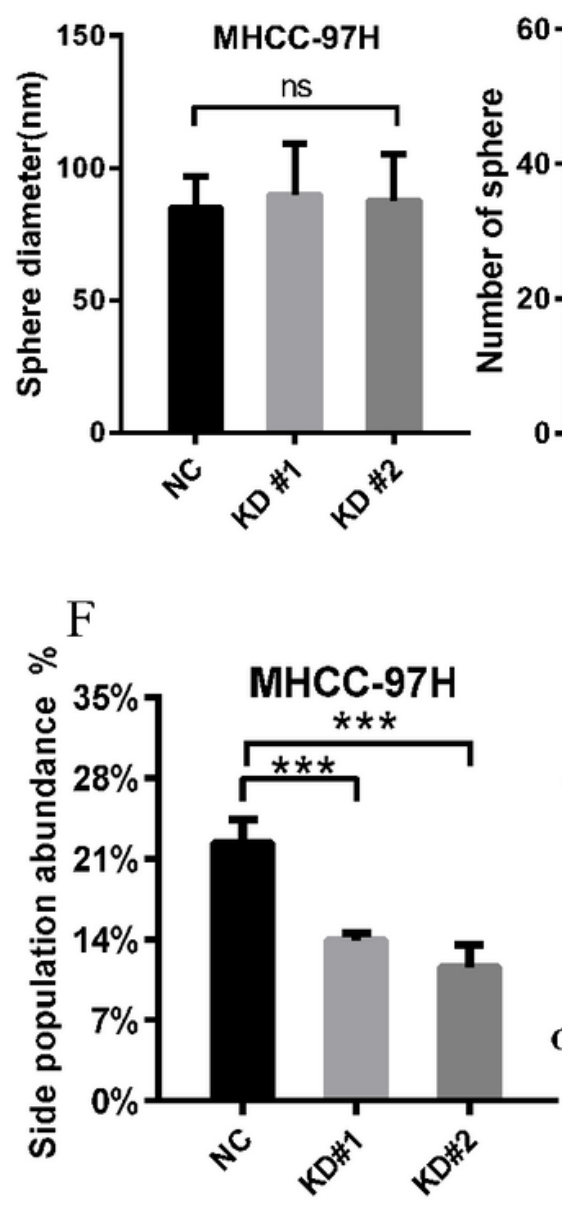

$\mathrm{G}$
$\mathrm{B}$

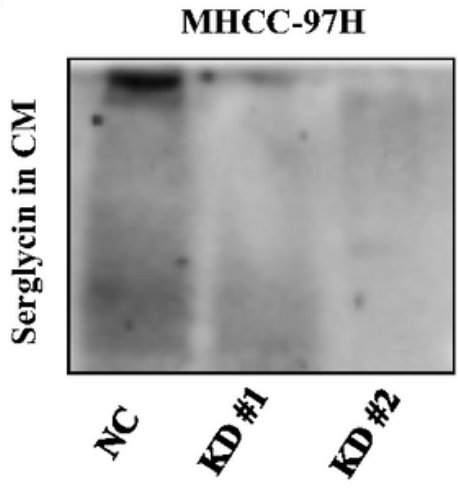

C

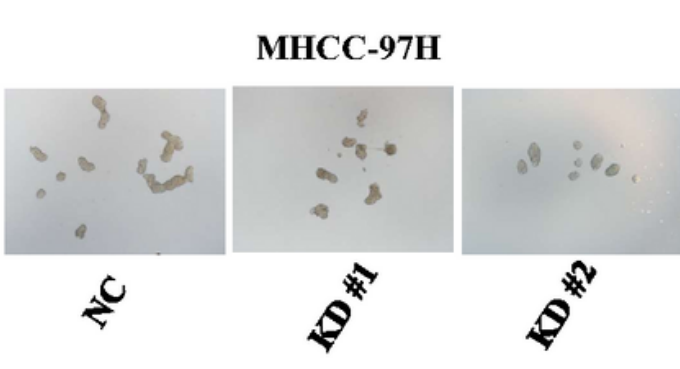

MHCC-97H

E

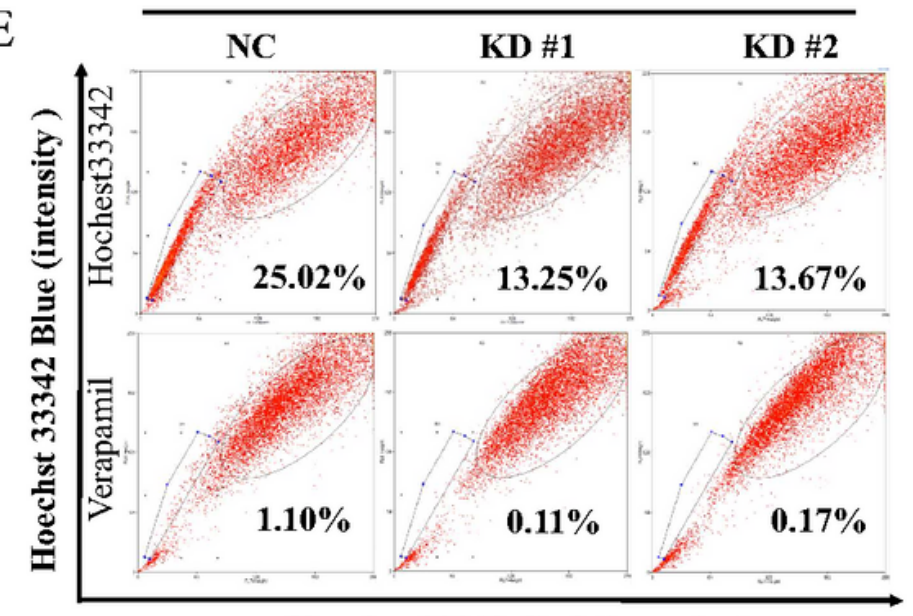

Hoechst 33342 Red (intensity)

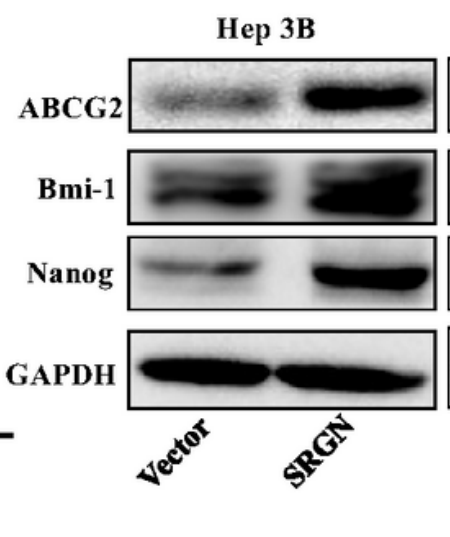

$\mathrm{H}$

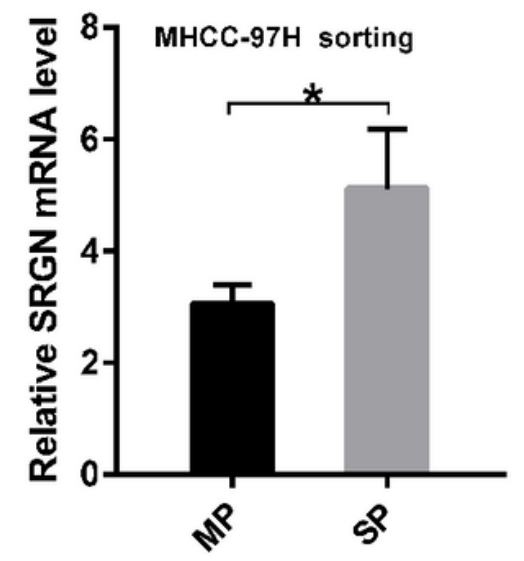

Figure 3 
SRGN is Significantly Associated with Stemness-Like Properties in HCC Cells (A) The knockdown and overexpression of SRGN in MHCC-97H cells were monitored by qPCR and immunoblotting. Number of suspended spheres following the knockdown of SRGN in MHCC-97H cells (C) and quantification of the tumorsphere size and number (D). (E, F) Side population (SP) cell assay. MHCC-97H cells with knockdown SRGN were treated with verapamil and Hoechst33342 dye and subjected to flow cytometry analysis. (G) Knockdown and overexpression of SRGN in HCC cells, and whole cell lysates were immunoblotted for ABCG2, Bmi-1, and Nanog at $72 \mathrm{~h}$ after transfection. $(\mathrm{H})$ The relative mRNA level of SRGN in the main population and side population of MHCC-97H cells was determined by q-PCR (normalized to GAPDH). ${ }^{\star} p<0.05,{ }^{\star} * \mathrm{p}<0.01$. 

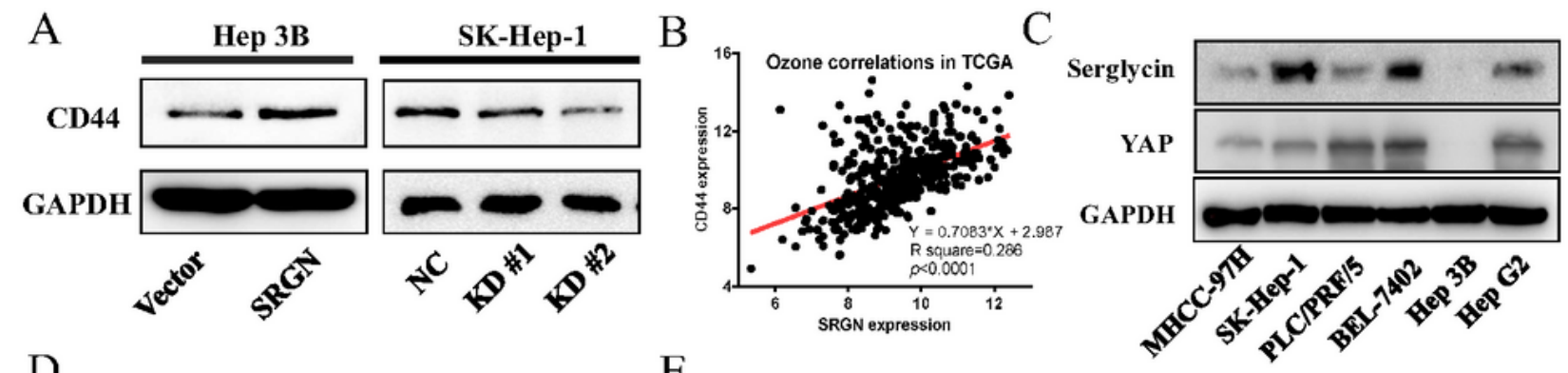

D

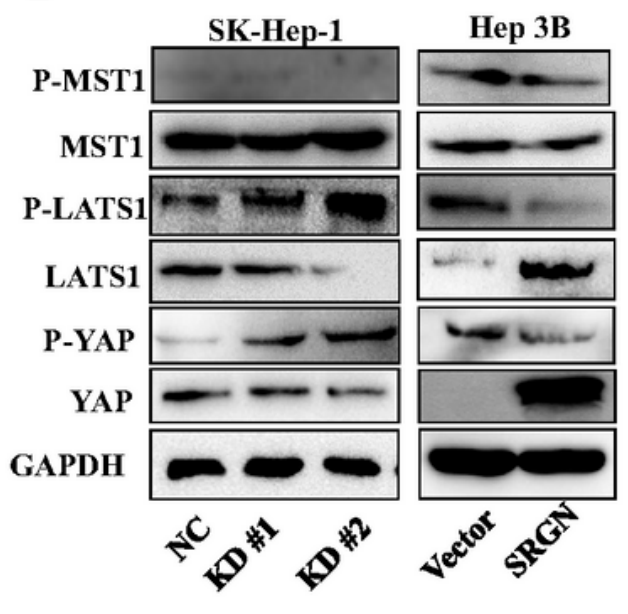

$\mathrm{E}$

SK-Hep-1

Нер 3B

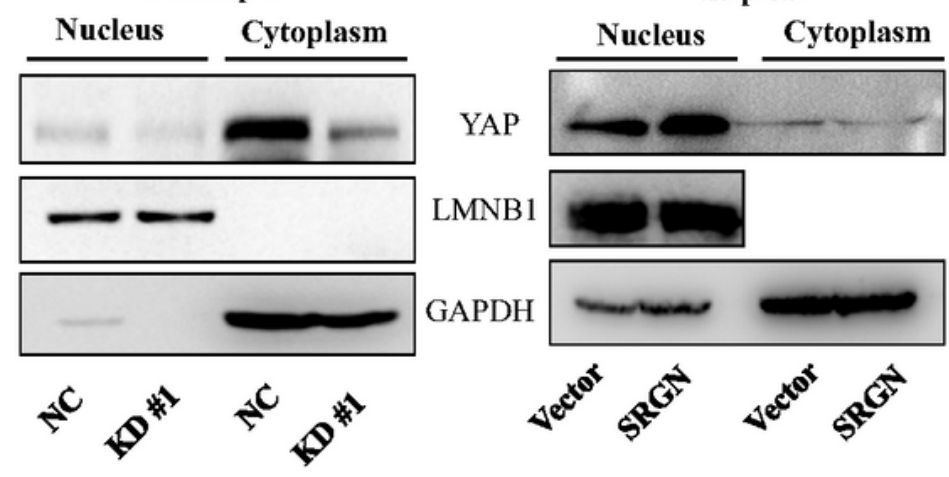

F

SK-Hep-1

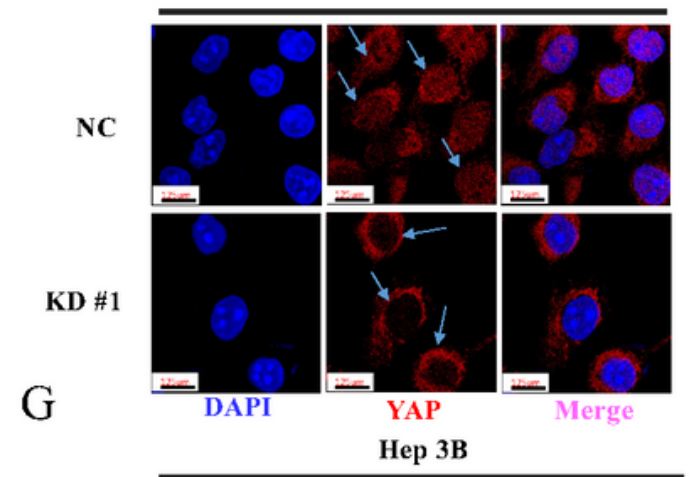

H

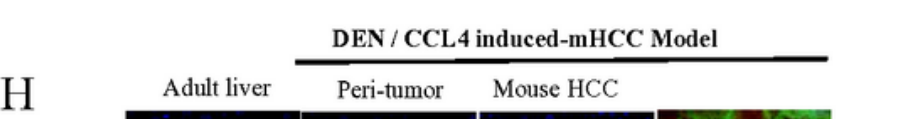

DAPI

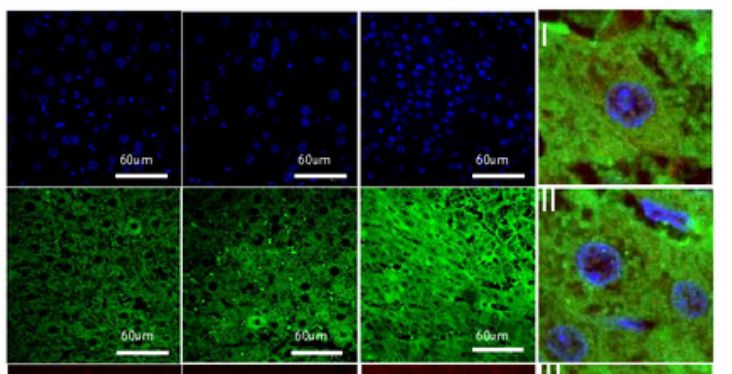

Yap

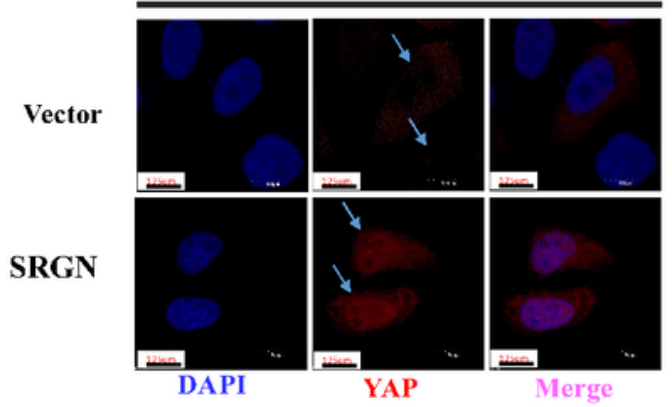

Serglycin

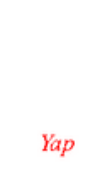

Merge

HE

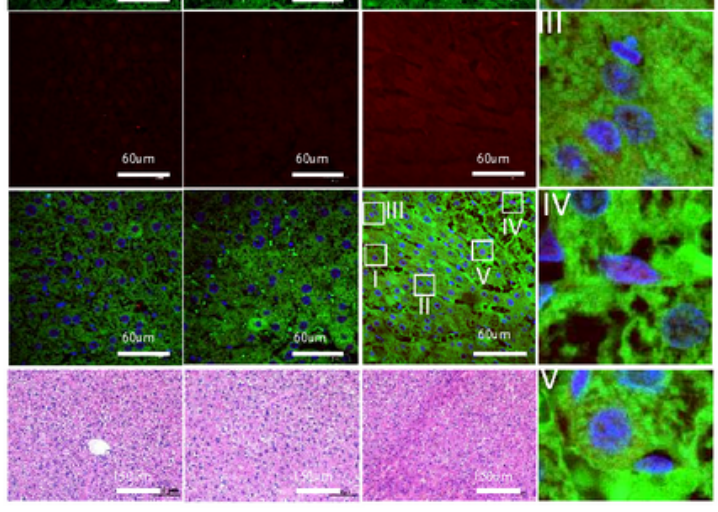

\section{Figure 4}

The CD44 Ligand SRGN Regulates the Nuclear Localization of the Hippo Pathway Effector YAP The expression levels of SRGN and CD44 were correlated in the HCC cell lines (A) and TCGA database (B). (C) The protein level was determined by Western blotting, which showed that SRGN and YAP levels were related to the expression in HCC cells. (D) Following the knockdown and overexpression of SRGN in HCC cells, the whole cell lysates were immunoblotted for MST1, P-LATS1, LATS1, P-YAP, and YAP at $72 \mathrm{~h}$ after 
transfection. Following the knockdown and overexpression of SRGN, the localization of YAP in SK-Hep-1 and Hep 3B was determined by immunoblotting $(E)$ and confocal immunofluorescence $(F, G)$. $(H)$ Localization of SRGN and Yap in the DEN/CCL4 induced mouse HCC Model by confocal immunofluorescence analysis.
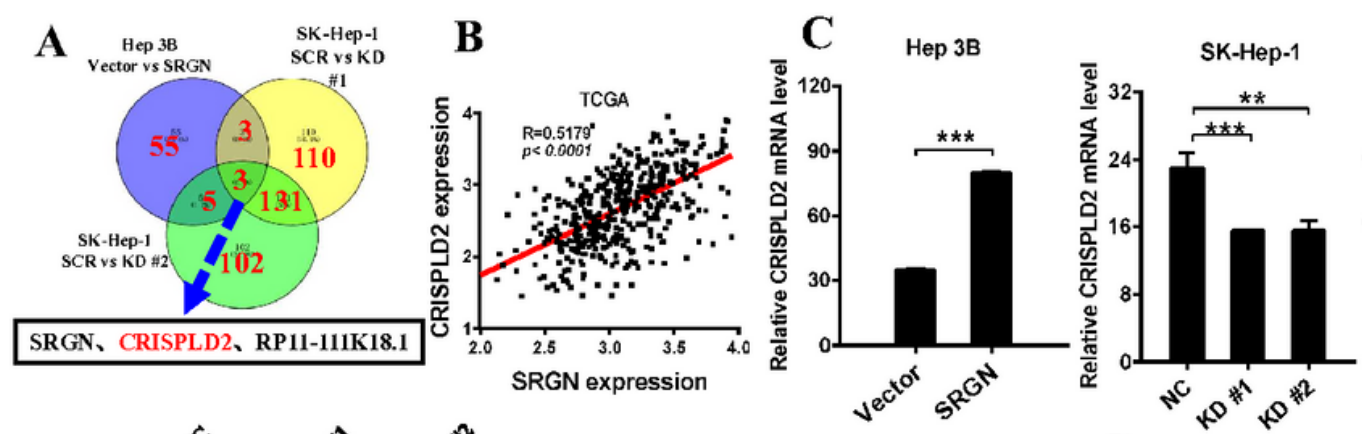

D
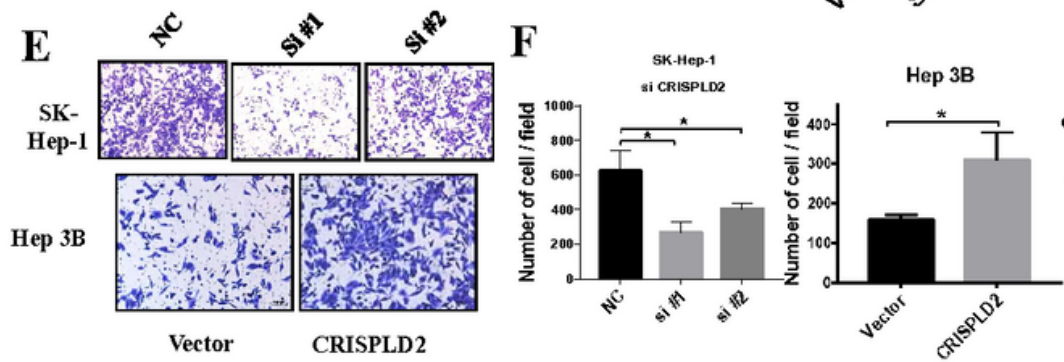

G

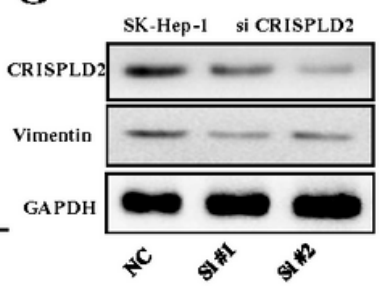

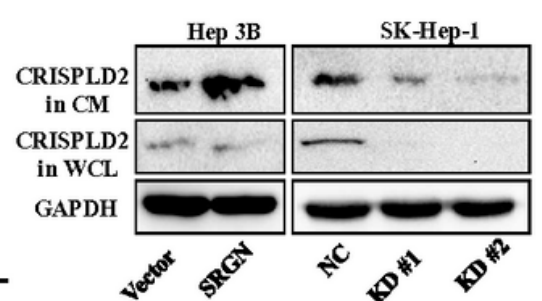

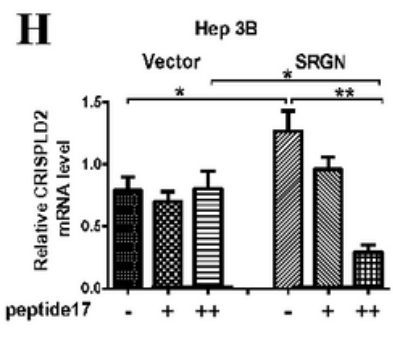

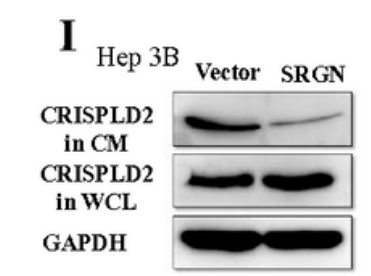

Peptide 17
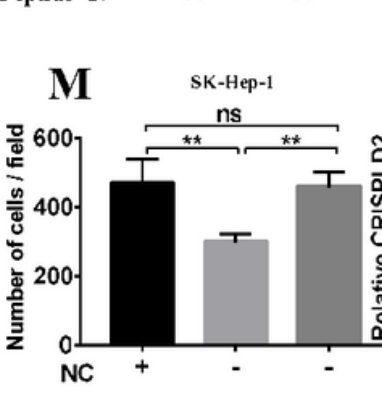

sh SRGN -

CRISPLD2 -

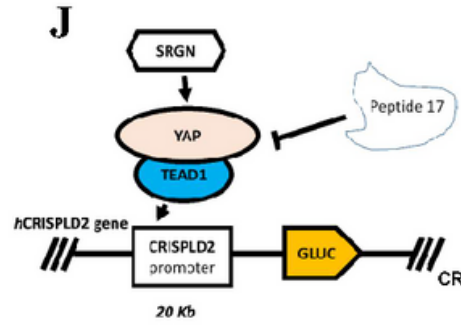

K

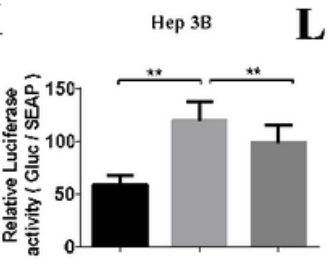

$\mathbf{L}$

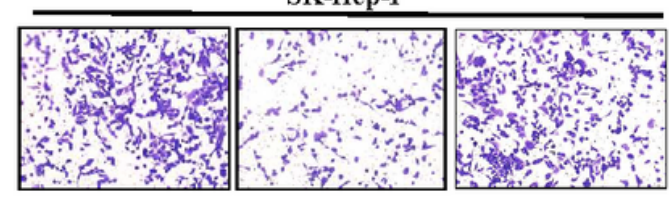

$\mathrm{NC}$

sh SRGN

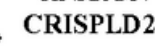

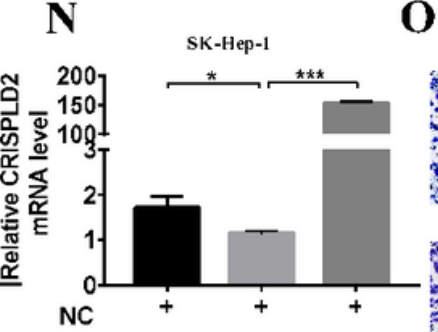

sh SRGN

CRISPLD2
$\mathbf{O}$ SRGN
Peptide $17 \div \quad+\quad+\quad$ CRISPLD2

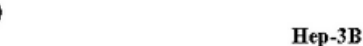

-3B
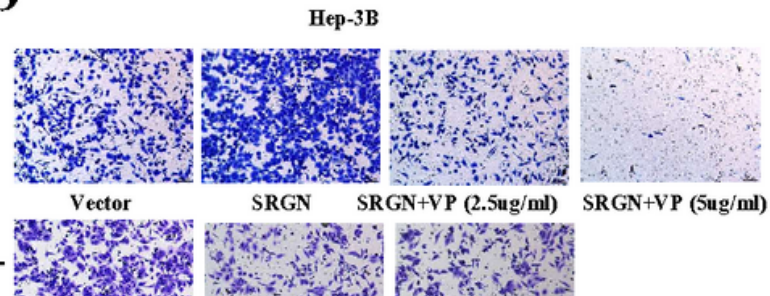

3 .

SRGN

SiCRISPLD2 \#N
Sols

SRGN

+siCRISPLD2

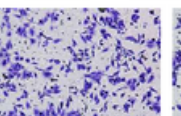

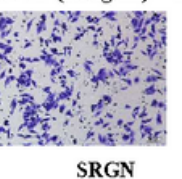

+ siCRISPLD2 \#2

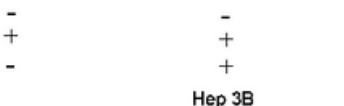

Hep 3B

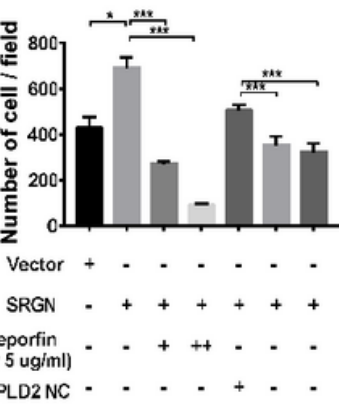

SICRISPLD2 $\#_{1} \cdot-\cdot \cdot \cdot+\cdot$

SICRISPLD2\#2 - . - . - . +

\section{Figure 5}

CRISPLD2 is an SRGN-Mediated HIPPO/YAP Pathway Downstream Effector Gene and Potentiates HCC Cell Motility Ability (A) Overlap of the differentially expressed genes from the overexpression and knockdown groups. SRGN expression was positively corrected with CRISPLD2 in the TCGA database (B) and HCC cells (C, D). The gain and loss of CRISPLD2 expression greatly affected the migration ability of cells in the Transwell assay (E), and CRISPLD2 expression in HCC cells was determined by qPCR (F) and 
immunoblotting (G). Overexpression of SRGN and treatment with peptide 17 and the mRNA expression (H) and protein levels (I) of CRISPLD2 in Hep 3B cells. (, $\mathrm{K}$ ) Hep 3B cells were treated with peptide 17 after SRGN overexpression. The relative luciferase activity was performed using the CRISPLD2 luciferase reporter. (L, M, N) Knockdown of SRGN and then overexpression of CRISPLD2 could reverse the cell migration ability of SK-Hep-1 cells compared with that of negative control cells. (I, P) Treatment with different doses of VP decreased the migration ability of SRGN in the HCC cells. Suppression CRISPLD2 can reverse the SRGN-promoting migration ability. ${ }^{*} p<0.05,{ }^{\star *} p<0.01,{ }^{\star *}{ }^{*} p<0.001$.
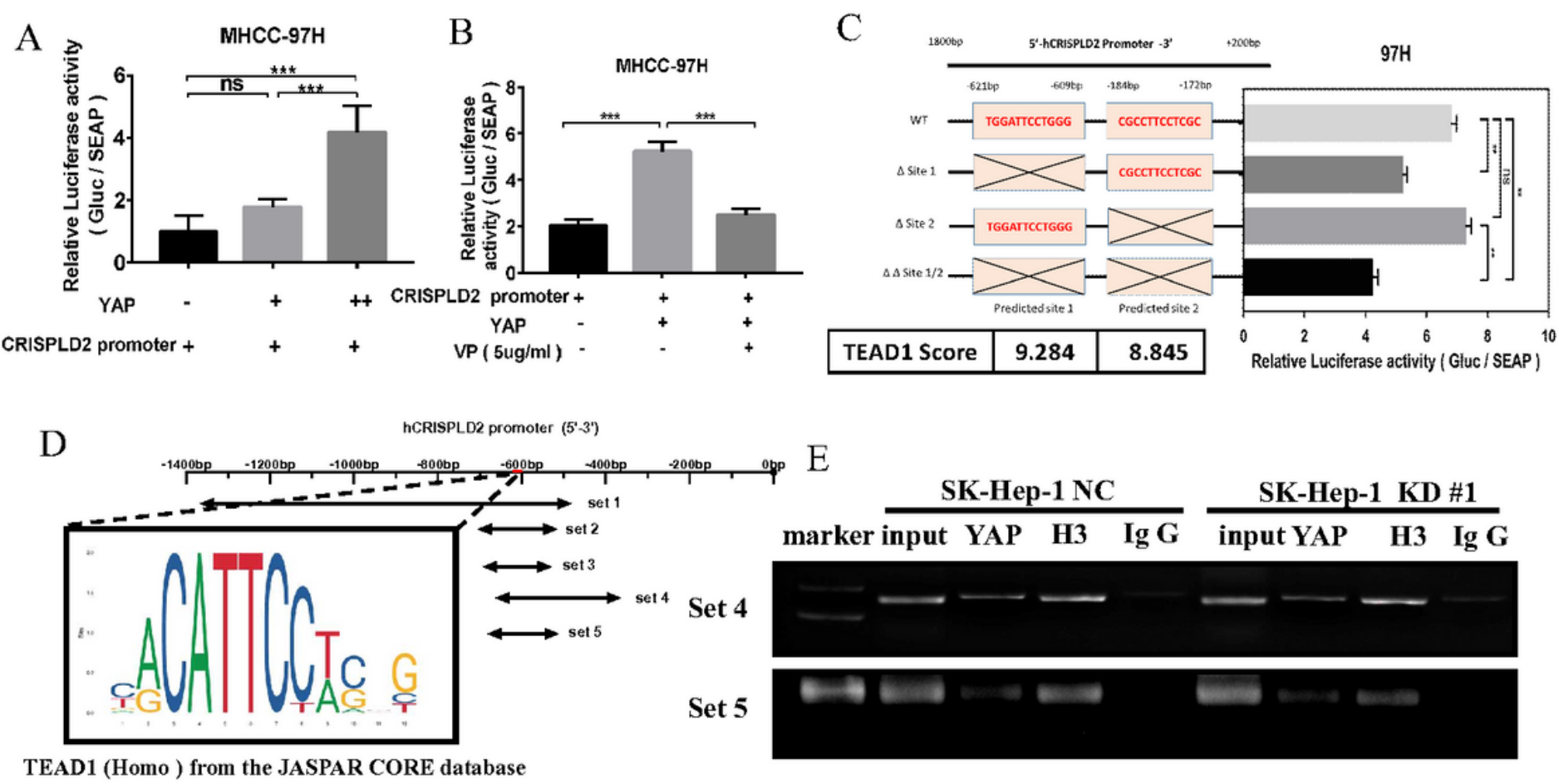

TEAD1 (Homo ) from the JASPAR CORE database

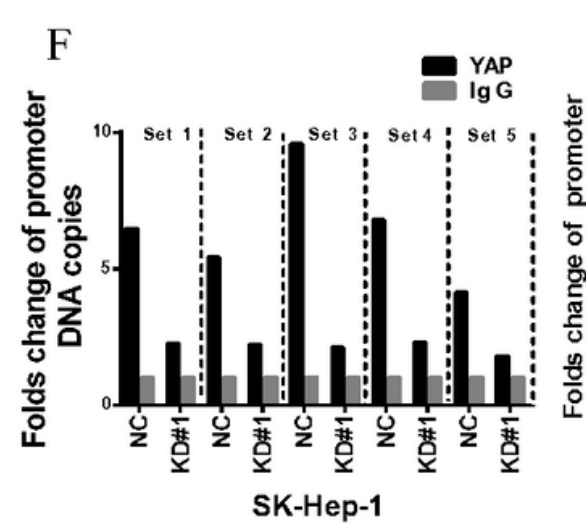

G $\quad \mathrm{H}$

CRISPLD2 Promoter TEAD1-binding Motif Genetic Conservation
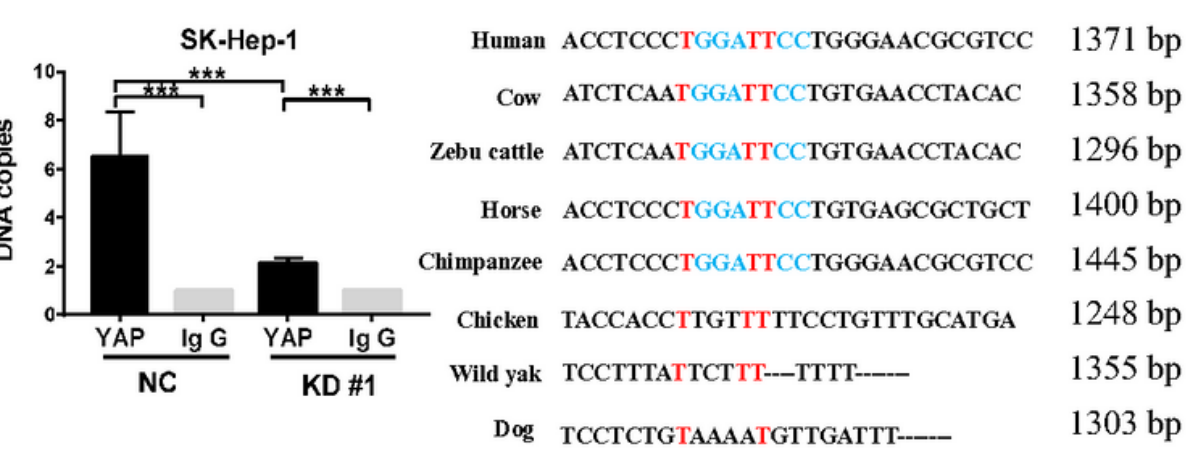

Figure 6

CRISPLD2 is a Novel YAP-TEAD1 Target Gene Dependent on SRGN. Luciferase activity of the CRISPLD2 promoter in YAP-overexpressing cells (A) and with verteporfin treatment (B). GLuc activities in buffers with a stabilizer. (C) Co-transcription factor YAP-TEAD1 binding site 1 motif in the CRISPLD2 promoter. 
(D) Schematic illustration of the binding sites of the CRISPLD2 promoter in the TEAD1 binding motif. (E, $\mathrm{F}, \mathrm{G}$ ) Analysis of the indicated knockout cells by ChIP and quantification of CRISPLD2 DNA using DNA agarose gel electrophoresis and qPCR. $(\mathrm{H})$ The conserved sequence of the CRISPLD2 motif in different species. ${ }^{* *} \mathrm{p}<0.01,{ }^{* * *} \mathrm{p}<0.001$.

A

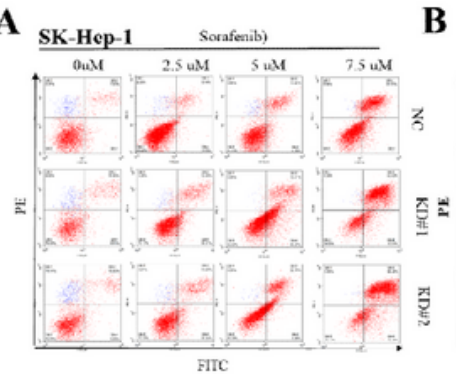

E

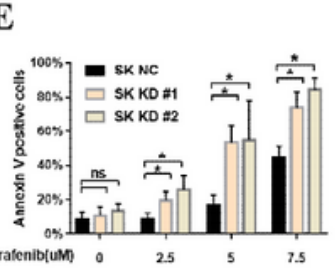

$\mathbf{J}$

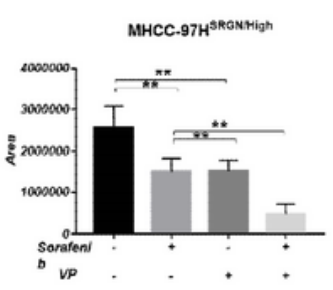

$\mathbf{K}$

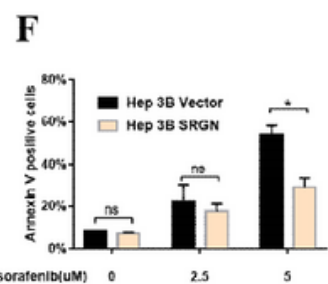

G

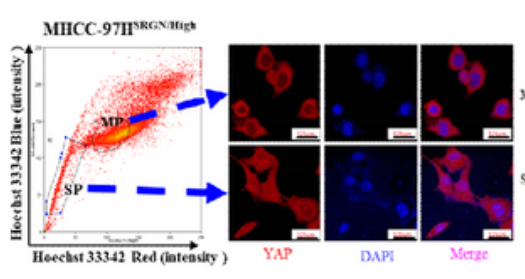

O

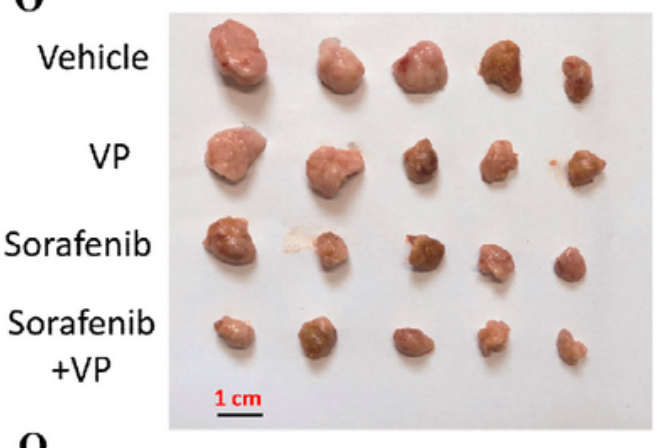

$\mathbf{P}$
C
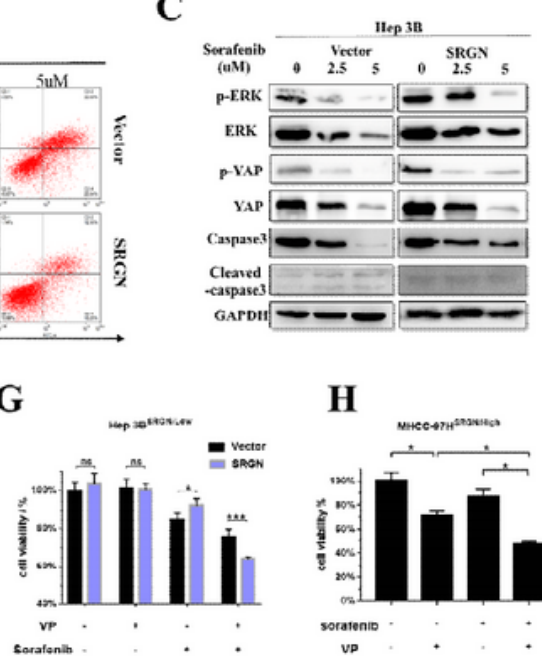

H
D
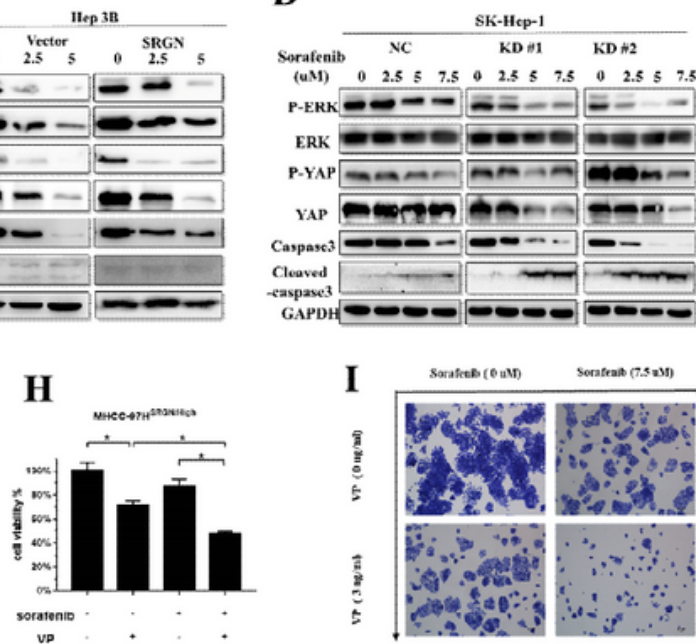

L

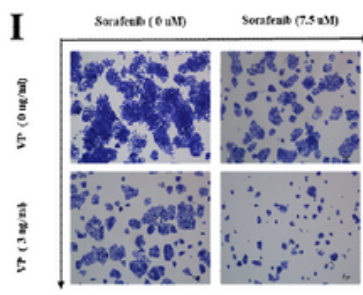

M

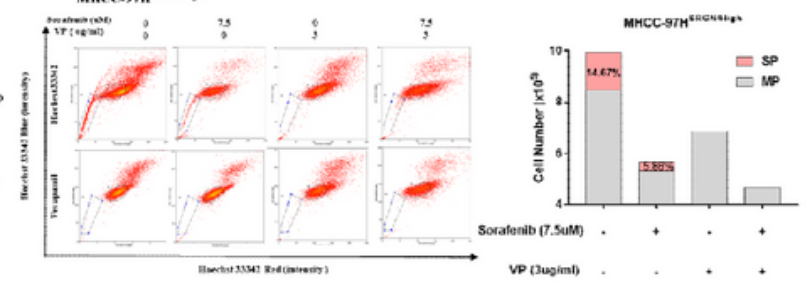

$\mathbf{R}$
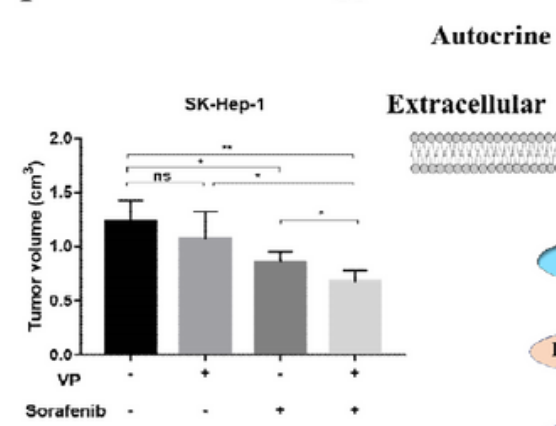

Q
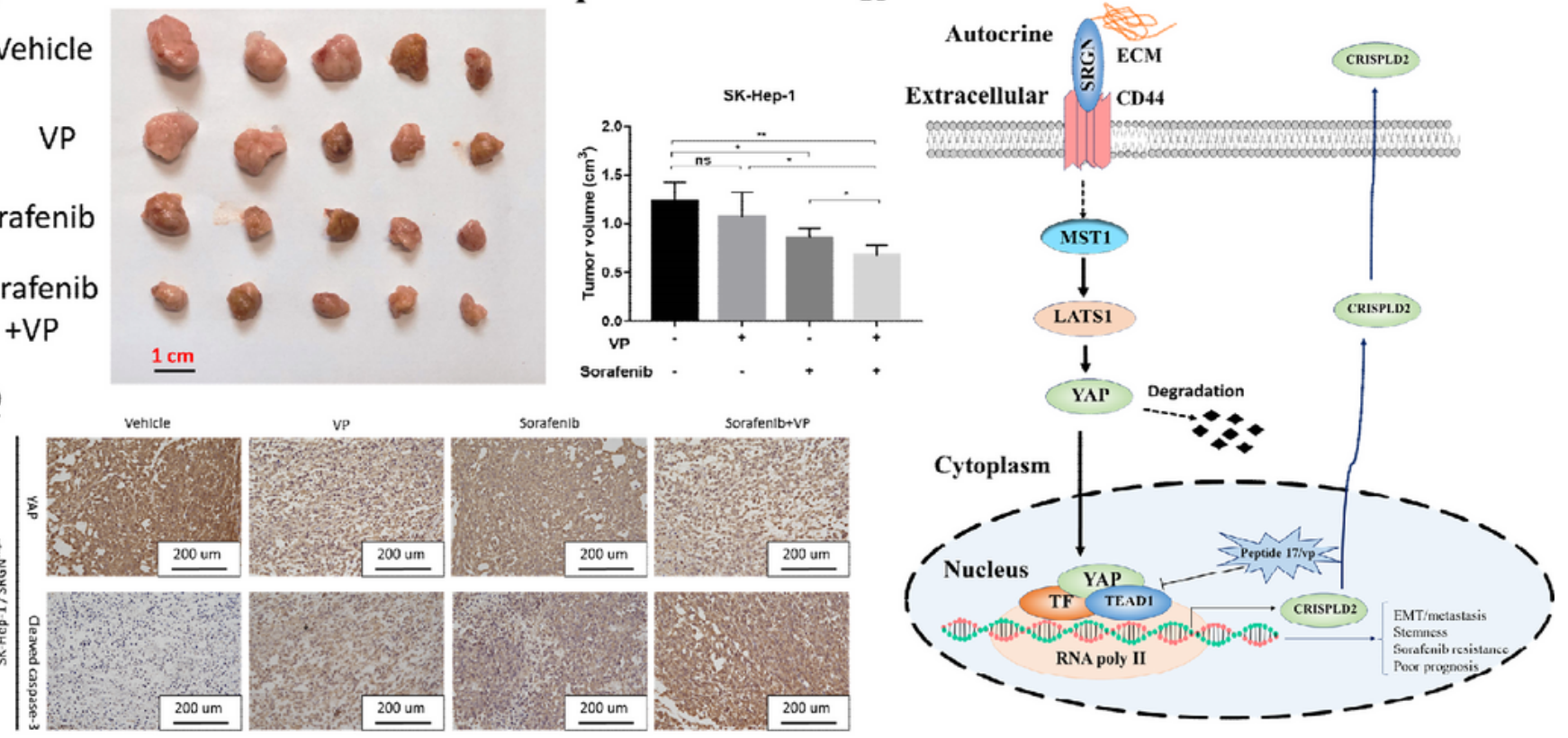

Figure 7 
Sorafenib Combined with Verteporfin Reverse the SRGN-Induced sorafenib resistance through Targeting YAP-Activated CSCs (A-D) SK-Hep-1 and Hep 3B cells were treated with sorafenib and analyzed by flow cytometry to evaluate apoptosis. (E, F) SK-Hep-1 and Hep 3B cells with SRGN knockdown and overexpression were treated with $0 \mathrm{nM}, 2.5 \mathrm{nM}, 5 \mathrm{nM}$, and $7.5 \mathrm{nM}$ sorafenib, and the P-ERK, ERK, P-YAP, YAP, caspase-3, cleaved-caspase-3 expression levels were analyzed by immunoblotting. GAPDH was used as the loading control. (G) Cell viability of Hep3B SRGN/low cells after treatment with VP and sorafenib. (H) Cell viability of MHCC-97HSRGN/High cells after treatment with VP and sorafenib. (I, J) MHCC97HSRGN/High cells were treated with VP and sorafenib for $48 \mathrm{~h}$ and then were subjected to colony formation. (K) SP detection in the sorted main population and side population. $(L, M)$ Localization of YAP in MHCC-97HSRGN/High MP and SP cells by confocal immunofluorescence analysis. SP detection in MHCC-97HSRGN/High cells that were treated with VP and sorafenib, followed by staining with Hoechst 33342 plus or minus verapamil. (O, P) Tumor volumes of SK-Hep-1SRGN/High xenografts treated with different combination schemes by intraperitoneal injection. (Q) Representative images of YAP and caspase-3 IHC staining of xenograft tumors. (R) Proposed model illustrating the tumorigenesis effect of SRGN in the pathogenesis of HCC. Superscript "SRGN/low" and "SRGN/High" represent low expression and high SRGN expression, respectively.

\section{Supplementary Files}

This is a list of supplementary files associated with this preprint. Click to download.

- SupplFig1.png

- Supplementary1.docx

- Supplementary2.docx

- Supplementary3.xls 